JOURNAL OF THE

AMERICAN MATHEMATICAL SOCIETY

Volume 13, Number 3, Pages 665-695

S 0894-0347(00)00338-6

Article electronically published on April 10, 2000

\title{
THE SIZE OF THE SINGULAR SET \\ IN MEAN CURVATURE FLOW \\ OF MEAN-CONVEX SETS
}

\author{
BRIAN WHITE
}

\section{INTRODUCTION}

In this paper, we study the singularities that form when a hypersurface of positive mean curvature moves with a velocity that is equal at each point to the mean curvature of the surface at that point.

It is most convenient to describe the results in terms of the level set flow (also called "biggest flow" I2]) of Chen-Giga-Goto CGG and Evans-Spruck ES. Under the level set flow, any closed set $K$ in $\mathbf{R}^{n+1}$ generates a one-parameter family of closed sets $F_{t}(K)(t \geq 0)$ with $F_{0}(K)=K$. If the boundary of $K$ is a smooth compact hypersurface, then so is the boundary of $F_{t}(K)$ for $t$ in some interval $[0, \epsilon)$, and for such $t$ 's the evolution coincides with motion by mean-curvature as defined classically by partial differential equations and differential geometry (as in [H1]). However, if $K$ is compact, then the boundary of $F_{t}(K)$ will necessarily become singular at some finite time.

Our goal is to show that the singular sets are necessarily quite small. This we do provided the initial set $K$ is compact and mean-convex in the sense that

$$
F_{t}(K) \subset \operatorname{interior}(K) \quad \text { for all } t>0 .
$$

In case $M=\partial K$ is a smooth hypersurface, we have the following equivalent characterization of mean-convexity: $K$ is mean-convex if and only if the mean-curvature of $M$ with respect to the inward unit normal is everywhere non-negative. Given a compact set $K$, let $\mathcal{K}$ be the region in spacetime swept out by the $F_{t}(K)$ :

$$
\mathcal{K}=\left\{(x, t) \in \mathbf{R}^{n+1} \times \mathbf{R}: t \geq 0, \quad x \in F_{t}(K)\right\} .
$$

A point $X=(x, t)$ in the boundary of $\mathcal{K}$ is called a regular point if

(1) $X$ has a neighborhood in which $\mathcal{K}$ is a smooth manifold-with-boundary, and

(2) the tangent plane to $\partial \mathcal{K}$ at $X$ is not horizontal (i.e., is not $\mathbf{R}^{n+1} \times[0]$ ).

Note that if $X=(x, t)$ is a regular point, then in a neighborhood of $x, F_{t}(K)$ is a smooth manifold-with-boundary in $\mathbf{R}^{n+1}$. A point $X=(x, t)$ in $\partial \mathcal{K}$ with $t>0$ that is not a regular point is called a singular point.

Received by the editors November 16, 1998 and, in revised form, March 15, 2000.

2000 Mathematics Subject Classification. Primary 53C44; Secondary 49Q20.

Key words and phrases. Mean curvature flow, mean convex, singularities.

The research presented here was partially funded by NSF grant DMS 9803403 and by a Guggenheim Foundation Fellowship. 
1.1. Theorem. Let $K$ be a compact mean-convex set in $\mathbf{R}^{n+1}$. Let $\operatorname{sing}(\mathcal{K})$ be the spacetime singular set (where $\mathcal{K}$ is as in $(*))$. Then the parabolic hausdorff dimension of $\operatorname{sing}(\mathcal{K})$ is at most $n-1$.

Corollary $([\mathrm{W} 3, \S 9])$. If

$$
(\operatorname{sing} \mathcal{K})_{t}=\{x:(x, t) \in \operatorname{sing}(\mathcal{K})\}
$$

is the singular set at time $t$, then

(1) $(\operatorname{sing} \mathcal{K})_{t}$ has hausdorff dimension $\leq n-1$ for every $t>0$ and

(2) for almost every $t>0$, the hausdorff dimension of $(\operatorname{sing} \mathcal{K})_{t}$ is $\leq n-3$.

In particular, if $n=2$, the surface is smooth for almost every $t$.

The parabolic hausdorff dimension of a set $S$ in $\mathbf{R}^{n+1} \times \mathbf{R}$ is the hausdorff dimension of $S$ with respect to the metric

$$
d((x, t),(y, \tau))=\max \left\{|x-y|,|t-\tau|^{1 / 2}\right\} .
$$

To understand what parabolic hausdorff dimension means, note that the time axis has parabolic dimension 2 (as it should for a parabolic problem). More generally, consider a smooth connected submanifold $N$ of spacetime whose ordinary dimension is $k$. If $N$ lies in some horizontal plane $\mathbf{R}^{n+1} \times[t]$, then its parabolic dimension is also $k$ (since the parabolic metric agrees with the euclidean metric on such horizontal planes). However, if $N$ does not lie in a horizontal plane, then its parabolic dimension is $k+1$.

Examples show that these results are in some sense optimal. Let $S$ be a unit $(n-1)$-sphere in $\mathbf{R}^{n+1}$. Let $K=K(r)$ be the set of points at distance $\leq r$ from $S$. (Thus if $n=2, K$ is a solid torus of revolution.) If $r>0$ is sufficiently small, then $K$ will be mean-convex. The flow preserves the rotational symmetry, so as soon as a singularity occurs (as it must), we get an entire $(n-1)$-sphere of singularities. (The singularity cannot occur on the axis of rotational symmetry because $F_{t}(K) \subset K$.) This shows that the $(n-1)$ in the theorem and in assertion (1) of the corollary cannot be replaced by any number $<n-1$.

However, in other ways the results are probably not optimal. For example, the theorem should hold for any "non-fattening" initial surface. Also, the singular set should have finite $(n-1)$-dimensional measure. Indeed, presumably it has nice rectifiability properties.

Behavior of surfaces near singularities. What does $M_{t}$ look like near a singularity? We have the following theorem:

1.2. Theorem. Let $\mathcal{M}$ be a mean curvature flow of compact mean-convex hypersurfaces in $\mathbf{R}^{n+1}$ with $n<7$. Let $\left(x_{i}, t_{i}\right)$ be any sequence of regular points of the flow that converge to a singular point $X=(x, t)$. Let $M(i)$ be obtained by translating $M_{t_{i}}$ by $-x_{i}$ and then dilating parabolically by the mean curvature of $M_{t_{i}}$ at $x_{i}$. Then a subsequence of the $M(i)$ converges smoothly on compact sets to a smooth convex surface $M^{\prime}$.

Furthermore, each tangent flow to $\mathcal{M}$ at $X$ is a shrinking sphere or cylinder. That is, if $\mathcal{M}^{\prime}$ is a tangent flow to $\mathcal{M}$ at $X$, then $M_{-1}^{\prime}$ is (after a rotation) of the form

$$
\mathbf{R}^{k} \times \partial \mathbf{B}^{n+1-k}(0, r)
$$

for some $k \in\{0,1, \ldots, n-1\}$. 
See [W5] for the proof, which uses results from this paper. If $n \geq 7$, we can also prove the same result, but only if the initial surface is smooth [W6].

Incidentally, the $M^{\prime}$ of Theorem 1.2 cannot be the cartesian product of the grim reaper curve with $\mathbf{R}^{n-1}$; see Corollary 12.5 .

Gerhard Huisken and Carlo Sinestrari [HS] have independently proved a result similar to 1.2 by a very different method. However, their proof is valid only up to the first occurrence of singularities. Also, in their result the sequence $\left(x_{i}, t_{i}\right)$ has to be chosen in a particular way.

Other ambient manifolds. All the results of this paper also apply when $K$ is a compact region of positive mean curvature inside an arbitrary smooth riemannian manifold. Only minor and straightforward modifications are necessary in the proofs.

In $\S 11$, we consider behavior as $t \rightarrow \infty$. In euclidean space, compact sets vanish in finite time under the level set flow. But this need not happen in other ambient spaces. We prove that if $K$ is a compact mean-convex subset of an $(n+1)$ dimensional riemannian manifold, then the boundary of $F_{t}(K)$ must converge nicely as $t \rightarrow \infty$ to a finite union of stable minimal hypersurfaces with small singular sets. In particular, if $n<7$, the boundary of $F_{t}(K)$ will eventually become regular and remain so.

Outline. In $\S 2$, we review basic concepts of level set flow and weak set flow. In $\S 3$, we prove some basic properties of level set flow for mean-convex sets. In $\S 4$, we prove, among other things, that there is a brakke flow of rectifiable varifolds associated to any level set flow of mean-convex surfaces. (When taking limits, such as blow-ups, of such flows, it is necessary to consider the brakke flow in order to keep track of multiplicities.)

Now at every spacetime point of a brakke flow, a subsequence of parabolic dilates of the flow about the point will converge to a tangent flow. Such tangent flows are analogous to tangent cones in minimal surface theory. In minimal surface theory, partial regularity results for minimal varieties are often proved by ruling out various kinds of singular tangent cones. Similarly, ruling out various kinds of tangent flows implies partial regularity results for mean curvature flows, as indicated in the following theorem. (The terms "static" and "quasistatic" will be defined in §5.)

1.3. Theorem $([\overline{\mathrm{W} 3}, \S 9])$. Suppose $\mathcal{M}$ is a brakke flow of $n$-dimensional rectifiable varifolds.

(1) If none of the tangent flows to $\mathcal{M}$ is a static plane of multiplicity $\geq 2$, then the spacetime singular set of $\mathcal{M}$ has parabolic hausdorff dimension $\leq n+1$.

(2) If in addition to (1), none of the tangent flows is a quasistatic plane of multiplicity $\geq 2$, then at each time the singular set has hausdorff dimension $\leq n-1$.

(3) If (in addition to (1) and (2)) none of the tangent flows is a static or quasistatic polyhedral cone, then the spacetime singular set has parabolic hausdorff dimension $\leq n-1$.

In $\S 3$, we prove if $K$ is mean-convex, then so is each $F_{t}(K)$. Furthermore, the boundary surfaces $M_{t}=\partial F_{t}(K)$ form a (possibly singular) foliation of $K$. It follows that each $M_{t}$ has a one-sided area minimization property. That property immediately implies (Theorem 3.8) that the $M_{t}$ cannot locally resemble a surface with multiplicity $\geq 3$. In particular, multiplicities higher than 2 cannot occur in any tangent flow to $\mathcal{K}$ if $K$ is mean-convex. 
The bulk of the paper is then devoted to excluding multiplicity 2 static and quasistatic planes. One crucial tool is an "expanding hole" theorem (Theorem 4.1), which says that if (in a certain ball) $M_{t}$ is suitably close to a multiplicity 2 disk with a hole, then (for a certain time) the hole will grow very rapidly.

In $\S 7$, we consider flows obtained by blowing up (in various ways) a mean-convex flow near a spacetime point of gaussian density $\leq 2$. In particular, we prove a Bernstein-type theorem that asserts under rather mild hypotheses that such a limit flow must consist of two parallel static planes.

This Bernstein-type theorem implies a "sheeting" theorem (Theorem 8.1): if a mean-convex flow is, in a given spacetime ball, weakly close to a smooth surface with multiplicity 2 , then in a smaller ball it must separate into two smooth sheets. This immediately implies (Corollary 8.5) that static multiplicity 2 planes do not occur as tangent flows.

In $\S 9$, we deduce from the sheeting theorem that no quasistatic multiplicity 2 tangent planes occur either.

Now if a tangent flow to a mean-convex flow is static or quasistatic, then (at any time $t<0$ ) the surface is actually a minimal cone. The mean-convexity of the flow implies that the cone is "one-sided minimizing", which implies that its singular set has codimension $\geq 7$. (To deduce the one-sided minimizing property of the limit flow from the one-sided minimizing property of the original flow requires that the convergence is rather nice. If the limit flow has multiplicity 1 almost everywhere, nice convergence follows from Brakke's regularity theorem [B, 6.10]. For multiplicity 2 limit flows, nice convergence follows from the "sheeting theorem" (Theorem 8.1). As already indicated, multiplicities higher than 2 cannot occur in limits of mean-convex surfaces.)

The general stratification (or dimension reducing) theory (Theorem 1.3, W3. $\S 9])$ then implies the desired bound on parabolic dimension of the singular set.

In $\S 11$, we consider ambient manifolds other than euclidean space.

In $\S 12$, we prove a few additional results about limits of mean-convex flows. The results are used in W5] to prove that for mean-convex surfaces in low dimensions, the only tangent flows are shrinking cylinders.

It is customary in the study of mean curvature flow to assume that the initial surface is smooth. Although that assumption is not necessary here, it does allow simpler proofs of some of the results. In such cases, the simpler proof is given first; readers willing to assume initial smoothness can then skip the rest of the proof.

Background material. For a general introduction to mean curvature flow from a measure-theoretic point of view, see [1], which includes a very nice description of Brakke's pioneering work [B]. Level set flow was introduced numerically in [OS], and then developped rigorously in ES] and CGG], but the approach introduced in [13] (also described in [11, §10] and [W2]) is more in line with this paper.

One of the main tools we use is the Brakke regularity theorem [B, 6.10]. Brakke's proof is quite involved. In another paper W4, we will give a rather simple proof of a version of Brakke's theorem. Though the result is not as strong as Brakke's, it nevertheless suffices for the applications in this paper.

See [H1] for a survey of many of the results about mean curvature flow that have been proved using partial differential equations and differential geometry. See [HS] and [St] for some other results about evolution of mean-convex surfaces.

The main results of this paper were announced in [W1. 


\section{Preliminaries}

2.1. Level set flow. If $K$ is a closed subset of $\mathbf{R}^{n+1}$ and $t \geq 0$, we let $F_{t}(K)$ denote the result of letting $K$ flow for time $t$ by the level set flow (also called "biggest flow" in [I3]). Level set flow has the following properties:

(1) If $t \mapsto M_{t}(0 \leq t \leq T)$ is a family of smooth compact hypersurfaces moving (classically) by mean-curvature, then

$$
F_{t}\left(M_{0}\right)=M_{t} \quad(0 \leq t \leq T) .
$$

(2) If $K$ and $K^{\prime}$ are disjoint closed sets with $K$ compact, then $F_{t}(K)$ and $F_{t}\left(K^{\prime}\right)$ are also disjoint, and $F_{t}\left(K \cup K^{\prime}\right)=F_{t}(K) \cup F_{t}\left(K^{\prime}\right)$.

(3) If $K \subset K^{\prime}$, then $F_{t}(K) \subset F_{t}\left(K^{\prime}\right)$.

(4) $F_{t+h} K=F_{t}\left(F_{h}(K)\right)$ and $F_{0}(K)=K$.

(5) $F_{t}$ commutes with translations.

These properties also imply:

(6) If $K$ is a compact subset of the interior of $K^{\prime}$, then $F_{t}(K)$ is contained in the interior of $F_{t}\left(K^{\prime}\right)$.

Proof. Suppose not. Then by translating $K$ slightly, we would get a set $K^{*}$ in $K^{\prime}$ for which $F_{t}\left(K^{*}\right) \not \subset F_{t}\left(K^{\prime}\right)$, contradicting (2).

Unfortunately, $F_{t}(K)$ does not depend continuously on $K$. For example, if $K_{i}$ is the union of two closed unit balls separated by distance $1 / i$, then

$$
\lim _{i} F_{t}\left(K_{i}\right) \neq F_{t}\left(\lim _{i} K_{i}\right)
$$

for $t>0$.

In other words, limits of level set flows need not be level set flows. For this reason, we also need weak set flows (called set-theoretic subsolutions in [11 §10] and in [13]).

2.2. Weak set flows. Any one-parameter family $K_{t}$ ( $t$ belonging to some interval $I$ ) of sets in space determines uniquely a subset

$$
\mathcal{K}=\left\{(x, t): x \in K_{t}\right\}
$$

of spacetime.

Definition. Suppose $\mathcal{K}$ is a closed subset of spacetime $\mathbf{R}^{n+1}$. Let

$$
t_{\text {initial }}=\inf \left\{t: K_{t} \neq \emptyset\right\}
$$

We say that $\mathcal{K}$ (or equivalently $t \mapsto K_{t}$ ) is a weak set flow (or weak flow for short) provided the following holds for every compact set $C \subset \mathbf{R}^{n+1}$, and for every $t \geq$ $t_{\text {initial }}$ and $h \geq 0$ : if $C$ is disjoint from $K_{t}$, then $F_{h}(C)$ is disjoint from $K_{t+h}$.

The definitions in [11, §10] and [13] (where such a $\mathcal{K}$ is called a "set-theoretic subsolution") and in [W2] are slightly different, but equivalent, to the one given here. In those papers, one only considers $C$ 's and $h$ 's such that $F_{s}(C)$ is a smooth embedded hypersurface for $0 \leq s \leq h$. The latter paper also considers motion of surfaces with boundary.

Suppose a sequence $\mathcal{K}_{i}$ of weak set flows converges (in the Hausdorff metric) to a limit set $\mathcal{K}$. (This convergence is equivalent to the following: (1) for every sequence $X_{i} \in \mathcal{K}_{i}$, all subsequential limits lie in $\mathcal{K}$, and (2) for every $X \in \mathcal{K}$, there is a sequence $X_{i} \in \mathcal{K}_{i}$ that converges to $X$.) It is not hard to prove that $\mathcal{K}$ is also 
a weak set flow. Furthermore, any sequence $\mathcal{K}_{i}$ of weak set flows (or even of closed sets) has a convergent subsequence.

2.3. Lemma. If $K$ is a closed set, then

$$
t \mapsto M_{t}=\partial F_{t}(K)
$$

is a weak set flow.

Proof. We must show that if a compact set $C$ is disjoint from $M_{t}$, then $F_{h} C$ is disjoint from $M_{t+h}$.

Consider first the case when $C$ is disjoint from $F_{t} K$. Then $F_{h}(C)$ is disjoint from $F_{h}\left(F_{t} K\right)=F_{t+h} K$ and therefore also disjoint from its boundary, namely $M_{t+h}$.

Now consider the case when $C$ is contained in the interior of $F_{t} K$. Then by $\S 2.1$ (6), $F_{h} C$ is contained in the interior of $F_{h}\left(F_{t} K\right)=F_{t+h} K$, and is therefore disjoint from its boundary, namely $M_{t+h}$.

Now if $C$ is an arbitrary compact set disjoint from $M_{t}$, then $C=C^{\prime} \cup C^{\prime \prime}$ where $C^{\prime}$ is disjoint from $F_{t} K$ and $C^{\prime \prime}$ is contained in the interior of $F_{t} K$. Now we have just shown that $F_{h}\left(C^{\prime}\right)$ and $F_{h}\left(C^{\prime \prime}\right)$ are disjoint from $M_{t+h}$. But $F_{h}(C)=$ $F_{h}\left(C^{\prime}\right) \cup F_{h}\left(C^{\prime \prime}\right)$, so $F_{h}(C)$ is also disjoint from $M_{t+h}$.

\section{MeAn-CONVEX SETS}

We say that a compact subset $K$ of $\mathbf{R}^{n+1}$ is mean-convex if, for some $\epsilon>0$,

$$
F_{h}(K) \subset \operatorname{interior}(K) \text { for } 0<h<\epsilon .
$$

Note that if $K$ is the closed region bounded by a compact, smooth embedded hypersurface whose mean curvature is everywhere positive, then $K$ is mean-convex (by $\S 2.1(1)$ ).

3.1. Theorem. Suppose $K$ is compact and mean-convex. Then

$$
F_{t+h}(K) \subset \operatorname{interior}\left(F_{t}(K)\right)
$$

for all $t+h>t \geq 0$. In particular, each $F_{t}(K)$ is mean-convex.

Proof. From $(*)$, we deduce (by $\S 2.1(4)$ and $(6)$ ) that

$$
F_{t+h}(K) \subset \operatorname{interior}\left(F_{t}(K)\right)
$$

for $0 \leq t$ and $0<h<\epsilon$. By induction we conclude that ( $\dagger$ ) holds with $h$ replaced by $i h$ for any $i=1,2,3, \ldots$ provided $0<h<\epsilon$. Equivalently, (†) holds for $0<h<i \epsilon$. Since this is true for all $i,(\dagger)$ holds for all $h>0$.

3.2. Theorem. If $K$ is compact and mean-convex, then every $x \in K$ is in the boundary of some $F_{t}(K)$. Thus, the boundaries of the $F_{t}(K)$ form a (possibly singular) foliation of $K$.

Proof. Let $T$ be the last time that $x \in F_{T}(K)$. Then $x \in \partial F_{T}(K)$. By 3.1, the $\partial F_{T}(K)$ are disjoint.

3.3. Corollary. If $K$ is compact and mean-convex, then

$$
F_{t}(\partial K)=\partial F_{t}(K) .
$$

In particular, the flow $t \mapsto F_{t}(\partial K)$ is non-fattening. (That is, for $t>0$, the interior of $F_{t}(\partial K)$ is empty.) 
Proof. Since

$$
\partial K \subset K,
$$

we have

$$
F_{t}(\partial K) \subset F_{t}(K)
$$

for all $t$.

Since $\partial K$ is disjoint from $F_{T}(K)$ for $T>0$, we have that

$$
F_{t}(\partial K)
$$

and

$$
F_{t}\left(F_{T}(K)\right)
$$

are disjoint for all $t$. Thus $F_{t}(\partial K)$ is disjoint from

$$
\bigcup_{T>0} F_{t}\left(F_{T}(K)\right)=\bigcup_{T>0} F_{t+T}(K) .
$$

But this set is the interior of $F_{t}(K)$. Thus we have shown:

$$
F_{t}(\partial K) \subset \partial F_{t}(K)=M_{t} .
$$

Since $t \mapsto F_{t}(\partial K)$ is the biggest flow and since $t \mapsto M_{t}$ is a weak flow (by Lemma $2.3)$, this implies that the two are equal.

Of course the boundary of a closed set has no interior, so the flow is non-fattening.

3.4. Lemma. Let $K$ be compact and mean-convex, and let $V$ be a locally area minimizing hypersurface (integral current) in the interior of $K$. Then the minimum distance from $V$ to $\partial K$ is attained only at the boundary of $V$.

Here " $V$ is locally minimizing" means that $V \backslash(\partial V)$ can be covered by balls $\mathbf{B}$ such that $V \cap \mathbf{B}$ is area minimizing.

Proof. Suppose not. Let $p \in \partial K$ and $q \in V$ realize the minimum distance. Then the tangent cone to $V$ at $q$ is a plane (because $V$ lies outside $\mathbf{B}(p,|q-p|)$ ), so $q$ is a regular point.

If $\partial K$ is smooth and of positive mean curvature, we get a contradiction of the classical maximum principle: translate $V$ by $p-q$ so that it just touches $\partial K$ at $p$.

If $\partial K$ is not smooth, note we have shown

$$
\operatorname{dist}(\partial K, V)<\operatorname{dist}(\partial K, V \backslash U)
$$

where $U$ is the set of regular non-boundary points of $V$. But this implies [W2, 4.1] that $\operatorname{dist}\left(F_{t}(\partial K), V\right)$ is non-decreasing on some interval $[0, \epsilon]$, which contradicts the fact that $\partial K$ is moving inwards.

3.5. One-sided Minimization Theorem. Suppose $K$ is a compact mean-convex set and $T>0$. Let $K_{t}=F_{t}(K)$.

(1) Suppose $V$ is a locally area minimizing hypersurface with boundary in $K_{T}$. If $V$ is contained in the interior of $K$, then it is contained in $K_{T}$.

(2) The boundary area of $K_{T}$ is less than or equal to the boundary area of $S$ if $K_{T} \subset S$ and $\bar{S} \subset$ interior $(K)$. 
Proof. To prove (1), suppose $V$ is not contained in $K_{T}$. Let $t$ be the first time that $\partial K_{t}$ touches $V$. Then for times $\tau$ just prior to $t$, we would have

$$
0<\operatorname{dist}\left(\partial K_{\tau}, V\right)<\operatorname{dist}\left(\partial K_{\tau}, \partial V\right)
$$

which (by the lemma) contradicts the mean-convexity of $K_{\tau}$ (Theorem 3.1). This proves (1).

To prove (2), consider first the case that $K$ has smooth boundary. Then if $S$ minimizes its boundary area subject to $K_{T} \subset S \subset K$, then $S$ will not touch $\partial K$, so $V=(\partial S) \backslash K_{T}$ will be locally area minimizing and therefore empty by assertion (1), proving the result.

If $\partial K$ is not smooth, the argument is slightly more complicated. Suppose $S$ is a counterexample to assertion (2). Let

$$
K^{\prime}=\{x \in K: \operatorname{dist}(x, \partial K) \geq \operatorname{dist}(S, \partial K)\}
$$

(where $\operatorname{dist}(S, \partial K)$ is the minimum distance from a point in $S$ to a point in $\partial K$ ). Thus

$$
K_{T} \subset S \subset K^{\prime} .
$$

We may now fix $K^{\prime}$ and suppose $S$ minimizes its boundary area subject to (*).

We claim that $V=(\partial S) \backslash\left(K_{T}\right)$ is locally area minimizing. For if $x \in V$, we can find a ball

$$
\mathbf{B}=\mathbf{B}(x, r) \subset K \backslash K_{T} .
$$

Let $W$ be the area minimizing surface with the same boundary as $V \cap \mathbf{B}$. By the convex hull property, $W$ is contained in $\mathbf{B}$. By Lemma 3.4, $W$ is also contained in $K^{\prime}$. Thus the area of $V \cap \mathbf{B}$ is less than or equal to the area of $W$, so $V \cap \mathbf{B}$ is minimizing. Thus $V$ is locally minimizing, which contradicts assertion (1).

3.6. Corollary. The area of $M_{t}=\partial F_{t}(K)$ is a decreasing function of $t$, and is finite for $t>0$.

3.7. Corollary. If $\mathbf{B}$ is a ball in $K$ and $V$ is an area minimizing surface with boundary in $\mathbf{B} \cap K_{T}$, then $V$ is contained in $\mathbf{B} \cap K_{T}$.

Proof. By the convex hull property, $V$ is contained in $\mathbf{B}$. Therefore by the theorem it is also contained in $K_{T}$.

3.8. Density-Bound Theorem. If $K$ is a compact mean-convex set and $\mathbf{B}(x, r)$ $\subset K$, then

$$
\mathcal{H}^{n}\left(M_{t} \cap \mathbf{B}(x, r)\right) \leq \mathcal{H}^{n}(\partial \mathbf{B}(x, r))=n \omega_{n} r^{n}
$$

where $M_{t}$ is the boundary of $F_{t}(K)$.

Proof. By 3.5, $M_{t}$ has less area than the boundary of $K_{t} \cup \mathbf{B}(x, r)$, which is obtained from $M_{t}$ by replacing $M_{t} \cap \mathbf{B}(x, r)$ by a portion of $\partial \mathbf{B}(x, r)$.

The following theorem says (in part) that if $M_{t} \cap \mathbf{B}(x, r)$ is close to a disk, then the multiplicity of that disk is $\leq 2+\epsilon$.

3.9. Multiplicity-Bound Theorem. Let $\mathbf{B}=\mathbf{B}(x, r)$ be a ball contained in a mean-convex set $K$. Let $K_{t}=F_{t}(K)$ and $M_{t}=\partial K_{t}$. Let $S$ be a slab in $\mathbf{B}$ of thickness $2 \epsilon r$ passing through the center of the ball:

$$
S=\{y \in \mathbf{B}: \operatorname{dist}(y, H)<\epsilon r\}
$$


where $H$ is a hyperplane passing through the center of the ball and $\epsilon>0$.

Suppose that $M_{t} \cap \mathbf{B}$ is contained in the slab $S$. Then $K_{t} \cap \mathbf{B} \backslash S$ consists of $k$ of the two connected components of $\mathbf{B} \backslash S$, where $k$ is 0 , 1, or 2. Furthermore:

$$
\operatorname{area}\left(M_{t} \cap \mathbf{B}\right) \leq(2-k+2 n \epsilon) \omega_{n} r^{n} .
$$

Proof. That $K_{t} \cap \mathbf{B} \backslash S$ consists of 0,1 , or both of the components of $\mathbf{B} \backslash S$ is trivial.

The one-sided minimization property implies that

$$
\mathcal{H}^{n}\left(M_{t}\right) \leq \mathcal{H}^{n}\left(\partial\left(K_{t} \cup S\right)\right) .
$$

Note that the boundary of $S$ consists of two $n$-disks of radius slighly less than $r$, together with a spherical band of area $\leq 2 n \omega_{n} \epsilon r^{n}$. Now $\partial\left(K_{t} \cup S\right)$ is obtained from $M_{t}$ by replacing $M_{t} \cap \mathbf{B}$ by $2-k$ of those disks together with a portion of the spherical band. Hence the desired estimate.

\section{EXPANDING HOLES}

A plane with a hole in it vanishes instantly under the level set flow (and therefore under any weak set-theoretic flow, or under any brakke flow). Here we show that if a set is flat enough, then a hole in it will grow rapidly for a while. (A related but more complicated result was proved by Brakke [B, 6.5].) The notion "flat enough" has to be specified with some care. Consider, for example, a catenoid: on any large scale it appears very flat, yet the hole does not grow.

Definition. If $S$ is a set in $\mathbf{R}^{n+1}, x$ is a point, and $r>0$, then the relative thickness of $S$ in $\mathbf{B}(x, r)$ is

$$
\operatorname{Th}(S, x, r)=\frac{1}{r} \inf _{|v|=1}\left(\sup _{y \in S \cap \mathbf{B}(x, r)}|v \cdot(y-x)|\right) .
$$

Thus, for example, $\operatorname{Th}(S, 0,1) \leq \epsilon$ if and only if, after a rotation, $S \cap \mathbf{B}(0,1)$ is contained in the slab

$$
\left\{y \in \mathbf{R}^{n+1}:-\epsilon \leq y^{n+1} \leq \epsilon\right\} .
$$

In the following theorem, $\delta$ controls how flat the surface is, and $A$ bounds (from below) how fast the hole is expanding.

4.1. Expanding Hole Theorem. For every $A<\infty$ there is a $\delta=\delta_{A}>0$ with the following property. If $\mathcal{K}$ is a weak set-theoretic flow and if $(x, t)$ is such that

$$
\operatorname{Th}\left(K_{t}, x, r\right)<\delta \quad \text { for } r \leq R
$$

and

$$
x \notin K_{t}
$$

then

$$
\operatorname{dist}\left(K_{t+r^{2}}, x\right) \geq A r \quad \text { for } 0 \leq r \leq \delta R .
$$

Proof. Suppose for a certain $A$ that the theorem is false. Then for every $\delta>0$ there is a weak flow $\mathcal{K}=\mathcal{K}^{\delta}$ for which (1) and (2) hold but (3) does not hold. By translating, we may assume $x=0$ and $t=0$. Let

$$
\rho=\rho^{(\delta)}=\inf \left\{r: \operatorname{dist}\left(K_{r^{2}}, 0\right) \leq A r\right\} .
$$


By (2), $\rho>0$. By scaling, we can assume that $\rho=1$. The failure of (3) implies that $\delta R \geq \rho=1$ and thus that $R \geq 1 / \delta$. Thus (1) implies

$$
\operatorname{Th}\left(K_{0}^{\delta}, 0, r\right) \leq \delta \quad(r \leq 1 / \delta) .
$$

Now let $\mathcal{K}$ be a subsequential limit of the $\mathcal{K}^{\delta}$ as $\delta \rightarrow 0$. Then

$$
\operatorname{Th}\left(K_{0}, 0, r\right)=0
$$

and

$$
\begin{aligned}
\operatorname{dist}\left(K_{r^{2}}, 0\right) & \geq A r \quad(r \leq 1), \\
\operatorname{dist}\left(K_{1}, 0\right) & =A .
\end{aligned}
$$

By (5), $K_{0}$ is contained in a plane $P$. Thus for every $t, K_{t}$ is a subset of $P$. By (6), $K_{1 / 2}$ is a proper subset of $P$. This implies that $K_{t}=\emptyset$ for $t>1 / 2$. But that contradicts (7).

Definition. If $\mathcal{K}$ is a weak flow such that

$$
K_{T} \subset K_{t}
$$

for all $T \geq t \geq t_{\text {initial }}$, we will say that $\mathcal{K}$ is a nested flow.

Of course if $K$ is mean-convex, then $t \mapsto F_{t}(K)$ is a nested flow.

4.2. Corollary. Suppose $\mathcal{K}$ is a nested set flow and $(x, t)$ is a point such that

$$
\limsup _{r \rightarrow 0} \operatorname{Th}\left(K_{t}, x, r\right)<\delta_{A}
$$

and such that $x \notin K_{t+h}$ when $h>0$. Then

$$
\liminf _{r \rightarrow 0} \frac{\operatorname{dist}\left(K_{t+r^{2}}, x\right)}{r} \geq A .
$$

Remark. Suppose $\mathcal{K}$ is not just a nested flow, but actually a mean-convex flow. Then the assumption $x \notin K_{t+h}$ is superfluous. For if $x \notin K_{t}$, the conclusion is vacuously true (indeed, the lim inf is infinite). And if $x \in K_{t}$, then the hypothesis about thickness implies that $x \in \partial K_{t}$, which implies (by mean-convexity) that $x \notin K_{t+h}$ for any $h>0$.

Proof. By hypothesis, there is an $R>0$ such that

$$
\operatorname{Th}\left(K_{t}, x, r\right)<\delta_{A} \quad \text { for } r \leq R .
$$

Thus for any $h>0$,

$$
\operatorname{Th}\left(K_{t+h}, x, r\right)<\delta_{A} \quad \text { for } r \leq R .
$$

Hence applying the theorem to the point $(x, t+h)$ gives

$$
\operatorname{dist}\left(K_{t+h+r^{2}}, x\right) \geq A r \quad \text { for } r \leq \delta_{A} R .
$$

Replacing $r$ by $\sqrt{r^{2}-h}$ gives

$$
\operatorname{dist}\left(K_{t+r^{2}}, x\right) \geq A \sqrt{r^{2}-h} \quad \text { for } 0 \leq \sqrt{r^{2}-h} \leq \delta_{A} R .
$$

Letting $h \rightarrow 0$ gives

$$
\operatorname{dist}\left(K_{t+r^{2}}, x\right) \geq A r \quad\left(r<\delta_{A} R\right) .
$$


4.3. Corollary. Let $\mathcal{K}$ be a nested weak set flow, and suppose

$$
\limsup _{r \rightarrow \infty} \operatorname{Th}\left(K_{0}, 0, r\right)<\delta_{A} .
$$

Then either

$$
\bigcap_{t \geq 0} K_{t} \neq \emptyset
$$

or

$$
\liminf _{r \rightarrow \infty} \frac{\operatorname{dist}\left(K_{r^{2}}, 0\right)}{r} \geq A .
$$

Proof. By hypothesis, there is an $R<\infty$ such that

$$
\operatorname{Th}\left(K_{0}, 0, r\right)<\delta_{A} \quad \text { for } r \geq R .
$$

If $\bigcap_{t \geq 0} K_{t}$ is empty, then for some sufficiently large $T$,

$$
K_{T} \cap \mathbf{B}(0, R)=\emptyset .
$$

Since $K_{T} \subset K_{0}$, (1) and (2) imply that

$$
\operatorname{Th}\left(K_{T}, 0, r\right)<\delta_{A} \quad(\forall r) .
$$

Thus, by the theorem,

$$
\operatorname{dist}\left(K_{T+r^{2}}, 0\right) \geq A r
$$

which immediately gives $(*)$.

\section{Associated BRAKKe Flows AND LIMIT FLOWS}

5.1. Theorem. Let $K$ be a compact mean-convex set. Then

$$
t \mapsto \mathcal{H}^{n}\left\llcorner\partial K_{t}\right.
$$

defines a brakke flow of integral varifolds (where $K_{t}=F_{t}(K)$ ).

Proof. According to [11, §11.4], this follows from non-fattening (Corollary 3.3) and finite area (Corollary 3.6).

Fix a compact mean-convex set $K$. Let

$$
\mathcal{K}=\bigcup_{t \geq 0}\left\{(x, t): x \in F_{t}(K)\right\} \subset \mathbf{R}^{n+1} \times \mathbf{R}
$$

be the level set flow (regarded as a subset of spacetime). Let $\mathcal{M}$ be the associated brakke flow. Such a pair $(\mathcal{M}, \mathcal{K})$ will be called a mean-convex flow.

Let $X=(x, t)$ be a point in spacetime with $t>0$, let $X_{i} \rightarrow X$, and let $\lambda_{i}$ be positive numbers tending to $\infty$. Let $\mathcal{M}_{i}$ (resp. $\mathcal{K}_{i}$ ) be the brakke flow (resp. weak set flow) obtained by translating $\mathcal{M}$ (resp. $\mathcal{K}$ ) in spacetime by $-X_{i}$ and then dilating parabolically by $\lambda_{i}$ (that is, by $\left.(y, \tau) \mapsto\left(\lambda_{i} y, \lambda_{i}^{2} \tau\right)\right)$. Such a sequence $\left(\mathcal{M}_{i}, \mathcal{K}_{i}\right)$ is called a blow-up sequence.

By the uniform density estimate 3.8 and the compactness theorem for brakke flows [11, §7], a subsequence of the $\mathcal{M}_{i}$ will converge to a brakke flow $\mathcal{M}^{\prime}$. By passing to a further subsequence, we may assume that the $\mathcal{K}_{i}$ converge to a limit weak set flow $\mathcal{K}^{\prime}$. 
We call such a pair $\left(\mathcal{M}^{\prime}, \mathcal{K}^{\prime}\right)$ a limit flow at $X$. If $X_{i} \equiv X$, then $\left(\mathcal{M}^{\prime}, \mathcal{K}^{\prime}\right)$ is called a tangent flow at $X$. In that case, $\left(\mathcal{M}^{\prime}, \mathcal{K}^{\prime}\right)$ must be "backward" self-similar [I4]:

$$
M_{-r^{2}}^{\prime}=(r)_{\#}\left(M_{-1}^{\prime}\right), \quad K_{-r^{2}}^{\prime}=(r)_{\#}\left(K_{-1}^{\prime}\right) .
$$

Thus if $\left(\mathcal{M}^{\prime}, \mathcal{K}^{\prime}\right)$ is a tangent flow, then the portions of $\mathcal{M}^{\prime}$ and of $\mathcal{K}^{\prime}$ up to time 0 are determined by $M_{-1}^{\prime}$ and $K_{-1}^{\prime}$, respectively.

Let $\left(\mathcal{M}^{\prime}, \mathcal{K}^{\prime}\right)$ be a limit flow. If

$$
M_{t}^{\prime}=M_{-1}^{\prime}, \quad K_{t}^{\prime}=K_{-1}^{\prime}
$$

for all $t$, we say that the limit flow is static. If $(*)$ holds for all $t<0$ but not for all $t$, we say the limit flow is quasistatic. Note if $\left(\mathcal{M}^{\prime}, \mathcal{K}^{\prime}\right)$ is static or quasistatic, then $M_{-1}^{\prime}$ is a stationary varifold.

5.2. Theorem. (1) The set of limit flows at $X$ is compact and is closed under translations (in spacetime) and under parabolic dilations.

(2) If $\left(\mathcal{M}^{\prime}, \mathcal{K}^{\prime}\right)$ is a limit flow to a mean-convex flow, then $\mathcal{K}^{\prime}$ is nested, i.e., $K_{t+h}^{\prime} \subset K_{t}^{\prime}$ for all $t$ and all $h \geq 0$.

Proof. Straightforward.

Note that (1) (which does not require mean-convexity) means that any limit of limit flows at $X$ is itself a limit flow at $X$.

The support of a brakke flow $\mathcal{M}$ of integral varifolds is the smallest closed subset spt $\mathcal{M}$ of spacetime such that for each $t$, the varifold $M_{t}$ is supported in $\left\{x \in \mathbf{R}^{n}\right.$ : $(x, t) \in \operatorname{spt} \mathcal{M}\}$. For times $t$ after the starting time of $\mathcal{M},(x, t)$ is in the support of $\mathcal{M}$ if and only if the gaussian density (see $\S 7$ ) of $\mathcal{M}$ at $(x, t)$ is $\geq 1$.

5.3. Theorem. Suppose $(\mathcal{M}, \mathcal{K})$ is a mean-convex flow and that $t>t_{\text {initial }}$. Then

$$
(x, t) \in \operatorname{spt} \mathcal{M} \Longleftrightarrow(x, t) \in \partial \mathcal{K} .
$$

Proof. One direction ( $\Longrightarrow$ ) follows immediately from the definition of support (and 5.1). To prove the other, suppose $(x, t)$ is in $\partial \mathcal{K}$, and let $r>0$. Since $\mathcal{K}$ is closed, $(x, t) \in \mathcal{K}$, so $x \in K_{t}$. But $x$ is not in the interior of $K_{t}$, since then $(x, t)$ would be in the interior of $\mathcal{K}$. Thus $x \in \partial K_{t}$.

Let $\tau<t$ be such that

$$
\mathbf{B}(x, r) \not \subset K_{\tau} .
$$

Then for all $T$ with $\tau<T<t$,

$$
x \in \operatorname{interior}\left(K_{T}\right) \subset \operatorname{interior}\left(K_{\tau}\right) .
$$

This together with $(*)$ implies that

$$
\mathcal{H}^{n}\left(\mathbf{B}(x, r) \cap \partial K_{T}\right)>0
$$

which implies that there is a point $y \in \mathbf{B}(x, r)$ such that

$$
y \in \operatorname{spt}\left(\mathcal{H}^{n}\left\llcorner K_{T}\right)=\operatorname{spt} M_{T}\right. \text {. }
$$

Hence $(y, T) \in \operatorname{spt} \mathcal{M}$. Thus there are spacetime points in spt $\mathcal{M}$ arbitrarily close to $(x, t)$. Since $\operatorname{spt} \mathcal{M}$ is closed, this means that $(x, t) \in \operatorname{spt} \mathcal{M}$.

5.4. Theorem. Let $\left(\mathcal{M}^{\prime}, \mathcal{K}^{\prime}\right)$ be a limit flow to a mean-convex flow. Suppose there is a hyperplane $P$ and a time $T \in(-\infty, \infty]$ such that for $t \leq T$, the support of $M_{t}^{\prime}$ 
is $P$. Then either

(1) $\mathcal{K}=H \times \mathbf{R}$ for some halfspace $H \subset \mathbf{R}^{n+1}$ and, for every $t, M_{t}$ is $\partial H$ with multiplicity 1 , or

(2) $\mathcal{K}=P \times\left(-\infty, T^{*}\right]$ for some hyperplane $P$ and some time $T^{*} \in(-\infty, \infty]$. For every $t<T^{*}, M_{t}$ is $P$ with multiplicity 2 and, for every $t>T^{*}, M_{t}=0$.

Proof. Note that the hypotheses imply that

$$
\operatorname{spt} \mathcal{M}^{\prime}=P \times\left(-\infty, T^{*}\right]
$$

for some $T \leq T^{*} \leq \infty$.

Let $\left(\mathcal{M}_{i}, \mathcal{K}_{i}\right)$ be a blow-up sequence converging to $\left(\mathcal{M}^{\prime}, \mathcal{K}^{\prime}\right)$. Then (by W3. $\S 7.3])$

$$
\limsup _{i}\left(\operatorname{spt} \mathcal{M}_{i}\right)=P \times\left(-\infty, T^{*}\right] .
$$

Since spt $\mathcal{M}_{i}=\partial \mathcal{K}_{i}$ by 5.3 , this means that the limit $\mathcal{K}^{\prime}$ of the $\mathcal{K}_{i}$ must either be

(1') $H \times \mathbf{R}$ for a halfspace $H \subset \mathbf{R}^{n+1}$ bounded by $P$, or

$\left(2^{\prime}\right)$ a subset of $P \times\left(-\infty, T^{*}\right]$.

In case $\left(1^{\prime}\right)$, by the multiplicity bound theorem 3.9 , the multiplicity is 1 .

In case $\left(2^{\prime}\right)$, by the same theorem, the multiplicity is $\leq 2$. If the multiplicity were 1 , then the convergence would be smooth (by the Brakke regularity theorem B, 6.10]) which would imply that $\mathcal{K}^{\prime}$ is a region bounded by $P \times \mathbf{R}$, contradicting $\left(2^{\prime}\right)$. Thus (in case $\left.\left(2^{\prime}\right)\right)$ the multiplicity must be 2 .

5.5. Theorem. Let $(\mathcal{M}, \mathcal{K})$ be a mean-convex flow, and let $\left(\mathcal{M}_{i}, \mathcal{K}_{i}\right)$ be a blow-up sequence converging to a limit flow $\left(\mathcal{M}^{\prime}, \mathcal{K}^{\prime}\right)$. Then

(1) The support of $\mathcal{M}^{\prime}$ is equal to $\partial \mathcal{K}^{\prime}$.

(2) $\partial \mathcal{K}_{i}$ converge (as sets) to $\partial \mathcal{K}^{\prime}$.

Proof. Let $X \in \partial \mathcal{K}^{\prime}$. Then there are $X_{i} \in \partial \mathcal{K}_{i}$ such that

$$
X_{i} \rightarrow X
$$

(This is just by the definition of convergence of closed sets.) But spt $\mathcal{M}_{i}=\partial \mathcal{K}_{i}$, so $X_{i}$ is in the support of $\mathcal{M}_{i}$. It follows (see [W3, $\left.\S 7.3\right]$ ) that $X$ is in the support of $\mathcal{M}^{\prime}$

Thus we have proved that

$$
\partial \mathcal{K}^{\prime} \subset \lim \partial \mathcal{K}_{i}=\operatorname{spt} \mathcal{M}^{\prime}
$$

Now trivially

$$
\operatorname{spt} \mathcal{M}^{\prime} \subset \mathcal{K}^{\prime}
$$

For almost every $X \in \operatorname{spt} \mathcal{M}^{\prime}$, one of the tangent flows at $X$ will be a static or quasistatic plane [W3, §9]. By Theorem 5.4, there will be points in the complement of $\mathcal{K}^{\prime}$ that are arbitrarily close to $X$. Hence every such $X$ will be in $\partial \mathcal{K}^{\prime}$.

We have shown that a dense subset of spt $\mathcal{M}^{\prime}$ is contained in $\partial \mathcal{K}^{\prime}$. It follows that all of spt $\mathcal{M}^{\prime}$ is contained in $\partial \mathcal{K}^{\prime}$. Combining this with $(*)$ gives the result.

Remark. It follows from 5.4 and 5.5 that a limit flow $\left(\mathcal{M}^{\prime}, \mathcal{K}^{\prime}\right)$ to a mean-convex flow is essentially determined by the set $\mathcal{K}^{\prime}$. For at almost every point $X$ of $\mathcal{M}^{\prime}$, there is a unique plane $P$ such that if $\left(\mathcal{M}^{\prime \prime}, \mathcal{K}^{\prime \prime}\right)$ is a tangent flow to $\left(\mathcal{M}^{\prime}, \mathcal{K}^{\prime}\right)$ at $X$, then $\mathcal{M}^{\prime \prime}$ is supported in $P \times \mathbf{R}$. But then according to Theorem 5.4 , both the plane and its multiplicity are determined by $\mathcal{K}^{\prime \prime}$. 


\section{ONE-SIDED MINIMIZING PROPERTY FOR LIMIT FLOWS}

If $\mathcal{M}$ is any brakke flow (defined for $t \geq \operatorname{some~initial~time~} t_{0}$ ), let $\operatorname{reg}(\mathcal{M})$ denote the set of points $X$ at which at least one tangent flow to $\mathcal{M}$ is a static multiplicity 1 plane. By the Brakke regularity theorem [B, 6.10], every $X \in \operatorname{reg}(\mathcal{M})$ has a neighborhood $U$ such that

$$
\operatorname{spt} \mathcal{M} \cap U
$$

is a smooth hypersurface in spacetime. Note (for the moment) we do not regard a point $X$ of multiplicity $>1$ as a regular point, even if $\operatorname{spt} \mathcal{M}$ is a smooth manifold near $X$.

We also let

$$
\begin{aligned}
\operatorname{sing} \mathcal{M} & =\left\{X=(x, t) \in \operatorname{spt} \mathcal{M}: X \notin \operatorname{reg} \mathcal{M}, \quad t>t_{0}\right\}, \\
(\operatorname{reg} \mathcal{M})_{t} & =\left\{x \in \mathbf{R}^{n+1}:(x, t) \in \operatorname{reg} \mathcal{M}\right\}, \\
(\operatorname{sing} \mathcal{M})_{t} & =\left\{x \in \mathbf{R}^{n+1}:(x, t) \in \operatorname{sing} \mathcal{M}\right\} .
\end{aligned}
$$

Suppose $U$ is a bounded open subset of spacetime and that

$$
\operatorname{spt} \mathcal{M} \cap \bar{U} \subset \operatorname{reg}(\mathcal{M}) \text {. }
$$

If $\mathcal{M}_{i}$ are brakke flows that converge to $\mathcal{M}$, then for sufficiently large $i$,

$$
\operatorname{spt} \mathcal{M}_{i} \cap \bar{U} \subset \operatorname{reg}\left(\mathcal{M}_{i}\right)
$$

and $\left(\operatorname{spt} \mathcal{M}_{i}\right) \cap U$ converges smoothly to $(\operatorname{spt} M) \cap U$ on compact subsets of $U$ (by Brakke's regularity theorem [B, 6.10]).

6.1. Theorem. Let $\left(\mathcal{M}^{\prime}, \mathcal{K}^{\prime}\right)$ be a limit flow to a mean-convex flow $(\mathcal{M}, \mathcal{K})$. Suppose $S$ is a compactly supported $(n-1)$-cycle such that one of the following holds at some time $t$ :

(1) $S$ is supported in $\left(\operatorname{reg} \mathcal{M}^{\prime}\right)_{t}$, or

(2) $S$ bounds a compactly supported rectifiable $n$-chain $Q$ in the support of $M_{t}^{\prime}$ and $\left(\operatorname{sing} \mathcal{M}^{\prime}\right)_{t}$ has $\mathcal{H}^{n}$ measure 0 .

Then there is a compactly supported rectifiable $n$-chain $V$ such that

(3) $\partial V=S$.

(4) No other surface bounded by $S$ has less mass than $V$ does.

(5) $V$ is supported in $K_{t}^{\prime}$.

Of course $S$ may bound more than one area-minimizing surface. The theorem asserts (under hypothesis (1) or (2)) that at least one of the area minimizing surfaces is supported in $K_{t}^{\prime}$. (Presumably they all are supported in $K_{t}^{\prime}$, but that assertion is not needed here.)

Proof. Let us first assume hypothesis (1). Let $\left(\mathcal{M}_{i}, \mathcal{K}_{i}\right)$ be a blow-up sequence converging to $\left(\mathcal{M}^{\prime}, \mathcal{K}^{\prime}\right)$. Then by the Brakke regularity theorem $[\mathrm{B}, 6.10],\left(M_{i}\right)_{t}$ converges smoothly to $M_{t}^{\prime}$ in a neighborhood of spt $S$. Thus we can find cycles $S_{i}$ supported in spt $M_{i}=\partial K_{i}$ such that the $S_{i}$ are converging (say in the flat norm) to $S$. Let $V_{i}$ be a mass minimizing $n$-chain bounded by $S_{i}$. By 3.7, $V_{i}$ is supported in $\left(K_{i}\right)_{t}$. By passing to a subsequence, we may assume that the $V_{i}$ converge to a limit $V$. Then $V$ is mass minimizing and $V$ is supported in $K_{t}^{\prime}$. This proves the theorem under hypothesis (1). 
Now suppose hypothesis (2) holds. Let

$$
U_{\epsilon}=\left\{x \in \operatorname{spt} M^{\prime}: \operatorname{dist}\left(x,\left(\operatorname{sing} \mathcal{M}^{\prime}\right)_{t}\right)<\epsilon\right\} .
$$

Now

$$
\operatorname{Mass}(Q\llcorner\cdot)
$$

is a finite Borel measure on spt $M_{t}^{\prime}$. Thus

$$
\operatorname{Mass}\left(Q\left\llcorner\left(\operatorname{sing} \mathcal{M}^{\prime}\right)_{t}\right)=\lim _{\epsilon \rightarrow 0} \operatorname{Mass}\left(Q\left\llcorner U_{\epsilon}\right) .\right.\right.
$$

But since $\left(\operatorname{sing} \mathcal{M}^{\prime}\right)_{t}$ has $\mathcal{H}^{n}$ measure 0 , it cannot support a non-zero flat $n$-chain. Thus (*) vanishes, so $Q_{\epsilon}:=Q-Q\left\llcorner U_{\epsilon} \rightarrow Q\right.$ (in the mass topology).

Consequently if we let $S_{\epsilon}=\partial Q_{\epsilon}$, then $S_{\epsilon}$ converges in flat norm to $S$. Now $S_{\epsilon}$ satisfies hypothesis (1), so it bounds a minimizing surface $V_{\epsilon}$ supported in $K_{t}^{\prime}$. Then a subsequence of the $V_{\epsilon}$ will converge to a limit $V$. Note that the limit must be area minimizing and must be supported in $K_{t}^{\prime}$.

6.2. Corollary. Suppose (for a particular $t)$ that $\left(\operatorname{reg} \mathcal{M}^{\prime}\right)_{t}$ is $\mathcal{H}^{n}$-almost all of spt $M_{t}^{\prime}$. Then $K_{t}^{\prime}$ has no "holes"; that is, the complement of $K_{t}^{\prime}$ has no bounded connected components.

Proof. Suppose the complement of $K_{t}^{\prime}$ had a bounded connected component $U$. Let $P$ be a hyperplane containing some points of $U$. Let $Q$ be the portion of $\partial U$ on one side of $P$. Then $P \cap U$ is the unique area minimizing surface having the same boundary as $Q$. Hence by Theorem 6.1, $P \cap \bar{U}$ is contained in $K_{t}^{\prime}$, a contradiction.

\section{LIMIT FLOWS WITH DENSITY $\leq 2$ AT INFINITY}

Let

$$
\mathcal{M}^{\prime}: t \mapsto M_{t}^{\prime} \quad\left(t \geq T_{0}\right)
$$

be a brakke flow of rectifiable $n$-varifolds in euclidean space. (Here we are following the terminology of [1]. In particular, $M_{t}^{\prime}$ denotes the radon measure in $\mathbf{R}^{n+1}$ associated to an $n$-dimensional varifold.) For spacetime points $X=(x, t)$ and "radii" $r \in\left(0, \sqrt{t-T_{0}}\right]$, we define the gaussian density ratio:

$$
\Theta\left(\mathcal{M}^{\prime}, X, r\right)=\frac{1}{(4 \pi)^{n / 2} r^{n}} \int_{y \in \mathbf{R}^{n+1}} e^{-|y-x|^{2} / 4 r^{2}} d M_{t-r^{2}}^{\prime} y .
$$

This quantity, introduced by Huisken [H2] (see also [St]) for classical surfaces and then used by Ilmanen [I4] for brakke flows, plays the same role in mean curvature flow that the ordinary density ratio plays in minimal surface theory; see W3. (The notation here differs slightly from that in [W3]; what is denoted here by $\Theta(\mathcal{M}, X, r)$ is written there as $\Theta(\mathcal{M}, X, \tau)$ where $\tau=r^{2}$. The notation here makes the analogy with minimal surfaces more apparent.)

In particular, for each $X, \Theta\left(\mathcal{M}^{\prime}, X, r\right)$ is monotonically increasing in $r$. This implies existence of the following limit, called the (gaussian) density of $\mathcal{M}^{\prime}$ at $X$ :

$$
\Theta\left(\mathcal{M}^{\prime}, X\right)=\lim _{r \rightarrow 0} \Theta\left(\mathcal{M}^{\prime}, X, r\right)
$$

At regular points $X$ of $\mathcal{M}^{\prime}$, the gaussian density is 1 . If $T_{0}=-\infty$, then the following limit also exists:

$$
\lim _{r \rightarrow \infty} \Theta\left(\mathcal{M}^{\prime}, X, r\right)
$$


This limit is independent of $X$. We call it the gaussian density at $\infty$ of $\mathcal{M}^{\prime}$ or simply the Density of $\mathcal{M}^{\prime}$, and denote it by $\Theta\left(\mathcal{M}^{\prime}, \infty\right)$.

If $\left(\mathcal{M}^{\prime}, \mathcal{K}^{\prime}\right)$ is a limit flow to $(\mathcal{M}, \mathcal{K})$ at $X$, then the Density of $\mathcal{M}^{\prime}$ is $\leq$ the gaussian density of $\mathcal{M}$ at $X$ :

$$
\Theta\left(\mathcal{M}^{\prime}, \infty\right) \leq \Theta(\mathcal{M}, X)
$$

If $\left(\mathcal{M}^{\prime}, \mathcal{K}^{\prime}\right)$ is actually a tangent flow to $(\mathcal{M}, \mathcal{K})$ at $X$, then

$$
\Theta(\mathcal{M}, X)=\Theta\left(\mathcal{M}^{\prime}, \infty\right)=\Theta\left(\mathcal{M}^{\prime}, 0, r\right)
$$

for all $r>0$.

Note that $\Theta\left(\mathcal{M}^{\prime}, X, r\right)$ need not be strictly increasing in $r$. Indeed, $\Theta\left(\mathcal{M}^{\prime}, X, r\right)$ is independent of $r$ if and only if $\mathcal{M}^{\prime}$ is backward self-similar about $X$.

(These facts about gaussian density ratios are proved in [14]; see also W3].)

Let $C$ be the class of limit flows $\left(\mathcal{M}^{\prime}, \mathcal{K}^{\prime}\right)$ such that

(1) The gaussian density at $\infty$ is $\leq 2$,

(2) $\left(\mathcal{M}^{\prime}, \mathcal{K}^{\prime}\right)$ is not a static or quasistatic multiplicity 2 plane.

Our goal in this section is to show that rather weak hypotheses on $\left(\mathcal{M}^{\prime}, \mathcal{K}^{\prime}\right) \in C$ imply that $\mathcal{M}^{\prime}$ is a pair of parallel static multiplicity 1 planes and that $\mathcal{K}^{\prime}$ is the region between them.

7.1. Theorem. Let $\left(\mathcal{M}^{\prime}, \mathcal{K}^{\prime}\right)$ be a flow in $C$. Then

(1) Every tangent flow to $\left(\mathcal{M}^{\prime}, \mathcal{K}^{\prime}\right)$ is also in $C$.

(2) $\left(\mathcal{M}^{\prime}, \mathcal{K}^{\prime}\right)$ is regular almost everywhere. Indeed, for every $t$, the singular set $\left(\operatorname{sing} \mathcal{M}^{\prime}\right)_{t}$ has hausdorff dimension $\leq n-1$.

Proof. Let $\left(\mathcal{M}^{\prime \prime}, \mathcal{K}^{\prime \prime}\right)$ be a tangent flow to $\left(\mathcal{M}^{\prime}, \mathcal{K}^{\prime}\right)$ at $X$. By monotonicity, $\left(\mathcal{M}^{\prime \prime}, \mathcal{K}^{\prime \prime}\right)$ has Density $\leq 2$. If the Density is $<2$, then of course it is not a static or quasistatic multiplicity 2 plane. Thus suppose $\left(\mathcal{M}^{\prime \prime}, \mathcal{K}^{\prime \prime}\right)$ has Density $=2$. Then by $(*)$ (applied to $\mathcal{M}^{\prime \prime}$ and $\mathcal{M}^{\prime}$ instead in place of $\mathcal{M}^{\prime}$ and $\mathcal{M}$ ),

$$
\Theta\left(\mathcal{M}^{\prime}, X\right) \geq 2 \text {. }
$$

Since $\Theta\left(\mathcal{M}^{\prime}, \infty\right) \leq 2$, monotonicity then implies that $\Theta\left(\mathcal{M}^{\prime}, X, r\right) \equiv 2$ for all $r$, which implies that $\mathcal{M}^{\prime}$ is backward self-similar about $X$. For notational convenience, let us assume that $X$ is the origin in spacetime. Then

$$
M_{t}^{\prime \prime}=M_{t}^{\prime}
$$

for all $t \leq 0$. Since $\left(\mathcal{M}^{\prime}, \mathcal{K}^{\prime}\right)$ is not a static or quasistatic multiplicity 2 plane, neither is $\left(\mathcal{M}^{\prime \prime}, \mathcal{K}^{\prime \prime}\right)$. This completes the proof of $(1)$.

It follows from (1) (and the definition of $C$ ) that no static or quasistatic planes of multiplicity $>1$ occur as tangent flows to $\left(\mathcal{M}^{\prime}, \mathcal{K}^{\prime}\right)$. Conclusion $(2)$ now follows from the general stratification theorem 1.3 (see also [W3, §9]).

Remark. This theorem does not depend on mean-convexity.

If $\left(\mathcal{M}^{\prime}, \mathcal{K}^{\prime}\right)$ is static or quasistatic, then $M_{t}^{\prime}$ and $K_{t}^{\prime}$ are independent of $t$ for all sufficiently small $t$. That is, there is a stationary varifold $M^{\prime}$, a closed set $K^{\prime}$, and a time $T$ such that $M_{t}^{\prime} \equiv M^{\prime}$ and $K_{t}^{\prime} \equiv K^{\prime}$ for $t \leq T$. Furthermore, the support of $M^{\prime}$ is equal to the boundary of $K^{\prime}$ (by Theorem 5.5).

7.2. Theorem. If $\left(\mathcal{M}^{\prime}, \mathcal{K}^{\prime}\right)$ is a static or quasistatic limit flow in the class $C$, then $M^{\prime}$ is a stable minimal hypersurface whose singular set has hausdorff dimension $\leq n-7$. In particular, $M^{\prime}$ cannot be a singular polyhedral surface. 
Proof. Let $C_{\text {stat }}$ be the set of $\left(M^{\prime}, K^{\prime}\right)$ corresponding to a static or quasistatic flow $\left(\mathcal{M}^{\prime}, \mathcal{K}^{\prime}\right)$ in the class $C$, and let $s$ be the supremum (over all $\left(M^{\prime}, K^{\prime}\right) \in C_{\text {stat }}$ ) of the dimension of the singular set of $M^{\prime}$. Now $C_{\text {stat }}$ is closed under translations, dilations, and taking tangent cones.

Claim. If $\left(M_{i}^{\prime}, K_{i}^{\prime}\right) \in C_{\text {stat }}$ converges to $\left(M^{\prime}, K^{\prime}\right) \in C_{\text {stat }}$, and if $x$ is a regular point of $\left(M^{\prime}, K^{\prime}\right)$, then for some $r>0$ and for all sufficiently large $i, \mathbf{B}(x, r)$ contains no singular points of $\left(M_{i}^{\prime}, K_{i}^{\prime}\right)$.

Proof of claim. Recall that for us (until the end of $\S 8$ ), "regular point" means "multiplicity 1 regular point". The claim then follows immediately from Allard's regularity theorem.

Hence standard dimension reducing implies that there is a cone $\left(M^{\prime}, K^{\prime}\right)$ in $C_{\text {stat }}$ such that

(1) The singular set of $M^{\prime}$ is an $s$-dimensional linear subspace $V$, and

(2) $\left(M^{\prime}, K^{\prime}\right)$ is invariant under translations by vectors parallel to $V$.

(The dimension reducing argument is presented nicely in appendix A of [SL]. The functions there are the density functions of the varifolds $M^{\prime}$.)

In particular, after a rotation $K^{\prime}$ is of the form $K^{*} \times \mathbf{R}^{s}$ where $K^{*}$ is a closed region in $\mathbf{R}^{n+1-s}$.

Note by Theorem 7.1(2) that $K_{t}^{\prime}$ has the one-sided minimizing property of Theorem 6.1 .

Now $s$ cannot be $n$, because $M^{\prime}$ would then be a plane, and none of the points would be singular.

Also, $s$ cannot be $n-1$, because then $M^{\prime}$ would be a union of $\geq 3$ halfplanes meeting along $V$. Note each halfplane has multiplicity 1, and thus it separates a portion of $K_{t}$ from the complement. Thus there would be a pair $H_{1}, H_{2}$ of the halfplanes such that the region between them is contained in the complement of $K^{\prime}$. Let $Q=\mathbf{B}^{n+1} \cap\left(H_{1} \cup H_{2}\right)$. Then (by Theorem 6.1) there is an area minizing surface bounded by $\partial Q$ (that is, by $\left.\left(\partial \mathbf{B}^{n+1}\right) \cap\left(H_{1} \cup H_{2}\right)\right)$ lying in $K_{t}^{\prime}$. But there is no such surface, so $s<n-1$.

Notice the 1-sided minimizing theorem 6.1 implies that the set of regular points of $M^{\prime}$ (or equivalently of $\partial K^{*}$ ) constitutes a stable minimal surface. That is, $\partial K^{*}$ is a stable minimal hypercone in $\mathbf{R}^{n+1-s}$ that is regular away from the origin (but singular at the origin). Since we have shown $s<n-1$, it follows from Simons' theorem ([SJ] or [SL $\S \mathrm{B} .2]$ ) that $s \leq n-7$.

7.3. Corollary. Let $\left(\mathcal{M}^{\prime}, \mathcal{K}^{\prime}\right)$ be a static flow in the class $C$. There is an $\eta>0$ such that if

$$
\liminf _{r \rightarrow \infty} \operatorname{Th}\left(K^{\prime}, 0, r\right)<\eta,
$$

then $M^{\prime}$ is a pair of parallel planes and $K^{\prime}$ is the region between them.

Proof. Since $M^{\prime}$ is stable and has a small singular set, and since $M^{\prime}$ is relatively flat at infinity, $M^{\prime}$ must be a union of planes [SS]. The assertion then follows from the multiplicity bound theorem 3.9. 
7.4. Separation Theorem. Let $\left(\mathcal{M}^{\prime}, \mathcal{K}^{\prime}\right)$ be a limit flow in the class $C$ and suppose

$$
P \subset \bigcap_{t} K_{t}^{\prime}
$$

for some hyperplane $P$. Suppose also that the complement of $\bigcap_{t} K_{t}^{\prime}$ contains points on each side of $P$. Then $\left(\mathcal{M}^{\prime}, \mathcal{K}^{\prime}\right)$ is static, and $K^{\prime}$ is the region between two parallel planes.

Proof. Fix a time $T$. Since $\left(\operatorname{sing} \mathcal{M}^{\prime}\right)_{T}$ has $\mathcal{H}^{n}$ measure 0 (by Theorem $7.1(2)$ ), almost every point in $P$ is either in $\left(\operatorname{reg} \mathcal{M}^{\prime}\right)_{T}$ or in the interior of $K_{T}^{\prime}$. Fix such a point $x \in P$. In either case (regular point or interior point), there will be a small ball $\mathbf{B}(x, r)$ such that at least one of the halves of $\mathbf{B}(x, r) \backslash P$ will be contained in $K_{T}^{\prime}$. Choose one such half, and let $H$ be the set of all points of $\mathbf{R}^{n+1}$ on the other side of $P$.

We will establish that $\partial\left(K_{T}^{\prime} \cup H\right)$ does not touch $P$. We already know that it does not touch $P$ at $x$. Let $y$ be any other point of $P$. Let $R=|x-y|$, and let

$$
Q=\left(\partial\left(K_{T}^{\prime} \cup H\right)\right) \cap \mathbf{B}(y, R) .
$$

Now by the one-sided minimization theorem 6.1 , there is an area minimizing surface $V$ with $\partial V=\partial Q$ such that $V$ lies in $K_{T}^{\prime}$. By the maximum principle for minimal surfaces, $V$ does not touch $P$ (except possibly at points of $\partial V$ ). In particular, $y \notin V$. Now $V, \mathbf{B}(y, R) \cap P$, and

$$
(\partial \mathbf{B}(y, R)) \cap K_{T}^{\prime} \backslash H
$$

all lie in $K_{T}^{\prime}$. Thus the region between them lies in $K_{T}^{\prime}$ (by 6.2). This proves that $K_{T}^{\prime} \cup H$ contains $y$ in its interior.

Since $y$ was arbitrary, it follows that $K_{T}^{\prime} \cup H$ contains all of $P$ in its interior. Thus $P$ is contained in the interior of $K_{t}^{\prime} \cup H$ for all $t<T$ as well. Since $T$ was arbitrary, it follows that this is true for all $t$.

Consequently, the brakke flow $\mathcal{M}$ splits into two connected components: one component lying in $H$ and the other in $H^{c}$. Each flow has Density $\geq 1$ (by monotonicity), and the sum of the Densities is $\leq 2$. Thus each Density is equal to 1 , which implies that each component is a static or quasistatic plane. By 5.4(1), each component is a static plane.

7.5. Bernstein-Type Theorem. There is an $\epsilon>0$ with the following property. If $\left(\mathcal{M}^{\prime}, \mathcal{K}^{\prime}\right)$ is a limit flow in the class $C$ such that

$$
\liminf _{r \rightarrow \infty} \frac{\operatorname{dist}\left(K_{r^{2}}^{\prime}, 0\right)}{r} \leq 1
$$

and

$$
\limsup _{r \rightarrow \infty} \operatorname{Th}\left(K_{-r^{2}}^{\prime}, 0, r\right)<\epsilon,
$$

then $\mathcal{M}^{\prime}$ is a pair of static, parallel, multiplicity 1 planes and $\mathcal{K}^{\prime}$ is the region between them.

Proof. We let $\epsilon$ be any positive number less than the $\eta$ of Corollary 7.3 and the $\delta_{2}$ of Corollary 4.3.

By (2),

$$
\operatorname{Th}\left(K_{0}^{\prime}, 0, r\right)<\epsilon<\delta_{2}
$$


for all $r$ greater than some $R<\infty$. Hence by Corollary 4.3 and (1),

$$
\bigcap_{t} K_{t}^{\prime}=S \neq \emptyset
$$

Let $\left(\mathcal{M}^{\prime \prime}, \mathcal{K}^{\prime \prime}\right)$ be a subsequential limit (as $\left.T \rightarrow \infty\right)$ of the flows obtained by translating $\left(\mathcal{M}^{\prime}, \mathcal{K}^{\prime}\right)$ by $(0,-T)$. Note that $\left(\mathcal{M}^{\prime \prime}, \mathcal{K}^{\prime \prime}\right)$ is static and that

$$
K_{t}^{\prime \prime}=S \quad(\forall t) .
$$

Since $K_{t}^{\prime \prime}$ does not depend on $t$, we drop the subscript. By (2),

$$
\limsup _{r \rightarrow \infty} \operatorname{Th}\left(K^{\prime \prime}, 0, r\right) \leq \epsilon<\eta .
$$

Now clearly $\mathcal{M}^{\prime \prime}$ has Density $\leq 2$. Thus either $\mathcal{M}^{\prime \prime}$ is a static multiplicity 2 plane or (by Corollary 7.3) $K^{\prime \prime}=S$ is the region between two parallel planes. Either way, $\bigcap_{t} K_{t}^{\prime}$ contains a plane $P$. Thus the result follows from the separation theorem 7.4 .

\section{The SHeEting THEOREM}

In this section and the next, we use the following conventions. If $X=(x, t)$ and $X^{\prime}=\left(x^{\prime}, t^{\prime}\right)$ are points in spacetime, we let $\operatorname{dist}\left(X, X^{\prime}\right)$ denote the parabolic distance:

$$
\operatorname{dist}\left((x, t),\left(x^{\prime}, t^{\prime}\right)\right)=\max \left\{\left|x-x^{\prime}\right|,\left|t-t^{\prime}\right|^{1 / 2}\right\} .
$$

(If $x$ and $y$ are points in space, $\operatorname{dist}(x, y)=|x-y|$ denotes the usual euclidean distance.) We also let

$$
\mathbf{R}^{n, 1}=\mathbf{R}^{n} \times \mathbf{R}
$$

denote spacetime and

$$
\mathbf{B}^{n, 1}(X, r)=\left\{Y \in \mathbf{R}^{n, 1}: \operatorname{dist}(Y, X)<r\right\}
$$

denote the spacetime ball of radius $r$ about $X$.

To avoid clutter, we will omit the superscripts when there is no ambiguity. The capital letters $X, Y, \ldots$ denote points in spacetime, so that (for example) $\mathbf{B}(X, r)$ denotes the spacetime ball of radius $r$ about $X$. Lower case letters $x, y, \ldots$ denote points in space, so that $\mathbf{B}(x, r)$ denotes the euclidean ball of radius $r$ about $x$.

If $\mathcal{S}$ and $\mathcal{S}^{\prime}$ are subsets of spacetime, then $\operatorname{HausDist}\left(\mathcal{S}, \mathcal{S}^{\prime}\right)$ denotes the hausdorff distance between $\mathcal{S}$ and $\mathcal{S}^{\prime}$. That is, HausDist $\left(\mathcal{S}, \mathcal{S}^{\prime}\right)$ denotes the larger of the two numbers:

$$
\sup _{X \in \mathcal{S}} \inf _{Y \in \mathcal{S}^{\prime}}\|X-Y\|
$$

and

$$
\sup _{X \in \mathcal{S}^{\prime}} \inf _{Y \in \mathcal{S}}\|X-Y\|
$$

In the following theorem, we say that $\left(\mathcal{M}^{\prime}, \mathcal{K}^{\prime}\right)$ is a homothetic image of $(\mathcal{M}, \mathcal{K})$ provided it is obtained from $(\mathcal{M}, \mathcal{K})$ by translating (in spacetime) and then parabolically dilating. 
8.1. Theorem. Let $H=\left\{(x, t) \in \mathbf{R}^{n+1,1}: x_{n+1}=0\right\}$. There is an $\epsilon>0$ with the following property. Suppose $\left(\mathcal{M}^{\prime}, \mathcal{K}^{\prime}\right)$ is either a homothetic image of the meanconvex flow $(\mathcal{M}, \mathcal{K})$ or a limit of such homothetic images (i.e., a limit flow), and supppose that

$$
\operatorname{HausDist}\left(\mathcal{K}^{\prime} \cap \mathbf{B}^{n+1,1}(0,4), H \cap \mathbf{B}^{n+1,1}(0,4)\right)<\epsilon .
$$

Then there exist $f, g: \mathbf{B}^{n, 1}(0,1) \rightarrow \mathbf{R}$ such that:

(1) $f \leq g$.

(2) $f$ and $g$ have $C^{2,1}$ norms $\leq \frac{1}{100}$.

(3) For $(x, t) \in \mathbf{B}^{n+1,1}(0,2)$ with $\left(x_{1}, \ldots, x_{n}, 0, t\right) \in \mathbf{B}^{n+1,1}(0,1)$,

$$
(x, t) \in \mathcal{K}^{\prime} \quad \Longleftrightarrow \quad f\left(x_{1}, \ldots, x_{n}, t\right) \leq x_{n+1} \leq g\left(x_{1}, \ldots, x_{n}, t\right) .
$$

(4) For each $x, f(x, t)$ and $g(x, t)$ are increasing and decreasing, respectively, as functions of $t$.

Remarks. (1) Since $\mathcal{K}^{\prime}$ is a nested flow (Theorem 5.2), the last conclusion (4) follows immediately from conclusions (1) and (3).

(2) Suppose $\left(\mathcal{M}^{\prime}, \mathcal{K}^{\prime}\right)$ is a homothetic image of the mean-convex flow $(\mathcal{M}, \mathcal{K})$, and let $\lambda$ be the dilation factor. Note that if the hypothesis $(*)$ holds for a small $\epsilon$, then $\lambda$ must be correspondingly large. That is, if $\left(\mathcal{M}_{i}, \mathcal{K}_{i}\right)$ is a sequence of homothetic images of $(\mathcal{M}, \mathcal{K})$ with dilation factors $\lambda_{i}$, and if

$$
\operatorname{HausDist}\left(\mathcal{K}_{i} \cap \mathbf{B}^{n+1,1}(0,4), H \cap \mathbf{B}^{n+1,1}(0,4)\right) \rightarrow 0,
$$

then $\lambda_{i} \rightarrow \infty$. For otherwise, a subsequence of the $\left(\mathcal{M}_{i}, \mathcal{K}_{i}\right)$ would converge to a homothetic image $\left(\mathcal{M}^{\prime}, \mathcal{K}^{\prime}\right)$ for which

$$
\mathcal{K}^{\prime} \cap \mathbf{B}^{n+1,1}(0,4)=H \cap \mathbf{B}^{n+1,1}(0,4) .
$$

But this contradicts the mean-convexity of $\mathcal{K}^{\prime}$.

(3) Of course the $f$ and $g$ of Theorem 8.1 must satisfy the non-parametric mean curvature flow equation:

$$
\frac{\partial u}{\partial t}=\sqrt{1+|\mathbf{D} u|^{2}} \mathbf{D}_{i}\left(\frac{\mathbf{D}_{i} u}{\sqrt{1+|\mathbf{D} u|^{2}}}\right) .
$$

Thus if $f$ and $g$ are equal at a point, by the maximum principle they must be identically equal. This can happen only if $\left(\mathcal{M}^{\prime}, \mathcal{K}^{\prime}\right)$ is a limit flow.

It is convenient to prove the following theorem, which immediately implies 8.1:

8.2. Theorem. Suppose $\left(\mathcal{M}_{i}, \mathcal{K}_{i}\right)$ is blow-up sequence such that

$$
\operatorname{HausDist}\left(\mathcal{K}_{i} \cap \mathbf{B}(0,4), H \cap \mathbf{B}(0,4)\right) \rightarrow 0 .
$$

Then for sufficiently large $i,\left(\mathcal{M}_{i}, \mathcal{K}_{i}\right)$ satisfies the conclusion of Theorem 8.1. Indeed, the corresponding functions $f_{i}$ and $g_{i}$ and all of their derivatives converge uniformly to 0 on $\mathbf{B}^{n, 1}(0,1)$.

The key is to show that $\mathcal{M}_{i} \cap \mathbf{B}(0,3)$ separates into two components. We do this by defining a set $S_{i}$, which we prove to be a separating hypersurface.

8.3. Lemma. Let $S_{i}$ be the set of points $(x, t)$ such that $x$ is the center of a closed ball in $\left(K_{i}\right)_{t}$ that contains two or more points of $\partial\left(K_{i}\right)_{t}$. For $X \in \mathcal{K}_{i}$, let

$$
R_{i}(X)
$$


be the infimum of numbers $R>0$ such that

$$
\mathbf{B}(X, R) \cap S_{i}
$$

is a smooth properly embedded hypersurface of $\mathbf{B}(X, R)$ and

$$
\mathbf{B}(X, R) \cap \partial \mathcal{K}_{i}
$$

has exactly two components, one on either side of $\mathbf{B} \cap S_{i}$. (If there are no such $R>0$, we let $R_{i}(X)=\infty$.)

Then $R_{i}(X)$ tends to 0 uniformly on $\mathbf{B}^{n+1,1}(0,3)$.

Proof of Lemma 8.3. Let

$$
L=\limsup _{i}\left(\sup \left\{R_{i}(X): X \in \mathcal{K}_{i} \cap \mathbf{B}^{n+1,1}(0,3)\right\}\right) .
$$

Of course we want to show that $L=0$. By passing to a subsequence, we can assume the limsup is actually a limit. It then follows that there is a sequence of points $X_{i} \in \mathcal{K}_{i} \cap \mathbf{B}^{n+1,1}(0,3)$ with

$$
R_{i}\left(X_{i}\right) \rightarrow L>0
$$

We wish to dilate $\mathcal{M}_{i}$ about $X_{i}$ just enough that it is appreciably different (in a suitable sense) from a static multiplicity 2 plane. We do this as follows. Fix a small $\epsilon>0$. (Any $\epsilon$ smaller than 1 , smaller than the $\delta=\delta_{2}$ of 4.1 , and smaller than the $\epsilon$ of 7.5 will do.) Let $\rho_{i}$ be the smallest number $\geq 0$ such that for all $r$ with $\rho_{i} \leq r \leq 1$, we have

$$
\operatorname{Th}\left(K_{t_{i}-r^{2}}, x_{i}, r\right)<\epsilon \text { and } \operatorname{dist}\left(K_{t_{i}+r^{2}}, x_{i}\right)<r
$$

where $\left(x_{i}, t_{i}\right)=X_{i}$. Equivalently, $\rho_{i}$ is the supremum of numbers $r \leq 1$ for which one of these two conditions (2) fails.

Now by $4.2, \rho_{i}>0$ for each $i$. Also, by the hypothesis of 8.2 ,

$$
\rho_{i} \rightarrow 0 \text {. }
$$

Now translate $\left(\mathcal{M}_{i}, \mathcal{K}_{i}\right)$ and $S_{i}$ in spacetime by $-X_{i}$ and dilate by

$$
(x, t) \mapsto\left(x / \rho_{i}, t / \rho_{i}^{2}\right)
$$

to get a new flow $\left(\mathcal{M}_{i}^{*}, \mathcal{K}_{i}^{*}\right)$ and a new set $S_{i}^{*}$. Of course $R_{i}\left(X_{i}\right)$ scales to $R_{i}^{*}=R_{i} / \rho_{i}$. By passing to a subsequence, we may assume that the $\left(\mathcal{M}_{i}^{*}, \mathcal{K}_{i}^{*}\right)$ and the $S_{i}^{*}$ converge to a limit flow $\left(\mathcal{M}^{*}, \mathcal{K}^{*}\right)$ and a limit set $S^{*}$.

By $(2),(3)$, and the definition of $\rho_{i}$, we have

$$
\operatorname{Th}\left(K_{-r^{2}}^{*}, 0, r\right) \leq \epsilon \text { for } r \geq 1
$$

and

$$
\operatorname{dist}\left(K_{r^{2}}^{*}, 0\right) \leq r \text { for } r \geq 1 .
$$

However, at least one of these inequalities is not strict when $r=1$. Thus $\mathcal{K}^{*}$ is not a static plane.

By Theorem $7.5, \mathcal{M}^{*}$ consists of two parallel multiplicity 1 planes and $\mathcal{K}^{*}$ is the slab between them.

In particular, $\mathcal{M}^{*}$ is completely regular. Thus (by Brakke's regularity theorem $[\mathrm{B}, 6.10])$, the $\mathcal{M}_{i}^{*}$ converge smoothly to $\mathcal{M}^{*}$, which implies that the $S_{i}^{*}$ converges smoothly on compact subsets of spacetime to the hyperplane in the middle of the slab $\mathcal{K}^{*}$. 
By (4) (with $r=1$ ), the two planes are within distance $\epsilon$ (which was chosen $<1$ ) of the origin. By the smooth convergence, for sufficiently large $i$,

$$
S_{i}^{*} \cap \mathbf{B}^{n+1,1}(0,1)
$$

is a smooth surface and

$$
\mathbf{B}^{n+1,1}(0,1) \cap \partial \mathcal{K}_{i}^{*}
$$

has exactly two components, one on either side of $S_{i}^{*}$. Thus (for such $i$ )

$$
R_{i}^{*} \leq 1
$$

which means that $R_{i}\left(X_{i}\right) \leq \rho_{i}$. Since $\rho_{i} \rightarrow 0$, this means $R_{i}\left(X_{i}\right) \rightarrow 0$, which completes the proof of the lemma.

Proof of Theorem 8.2. Let

$$
\tilde{R}_{i}=\sup \left\{R_{i}(X): X \in \mathcal{K}_{i} \cap \mathbf{B}^{n+1,1}(0,3)\right\}
$$

where $R_{i}(X)$ is as in the lemma. Then by the lemma, $\tilde{R}_{i} \rightarrow 0$. Now when $\tilde{R}_{i}<\infty$, $S_{i} \cap \mathbf{B}^{n+1,1}(0,3)$ is a smooth properly embedded submanifold of $\mathbf{B}^{n+1,1}(0,3)$. Thus $S_{i}$ divides $\mathbf{B}^{n+1,1}(0,3)$ into two disjoint open subsets (not necessarily connected), each of which is bounded by $S_{i}$.

(This last assertion is standard, elementary topology. The division is accomplished as follows. Any two points in $\mathbf{B}^{n+1,1}(0,3) \backslash S_{i}$ can be joined by a path in $\mathbf{B}^{n+1,1}(0,3)$ that crosses $S_{i}$ transversely. Call the two points equivalent if the path crosses $S_{i}$ an even number of times. This does indeed define an equivalence relation, and obviously there are exactly two equivalence classes, namely the two desired disjoint open sets.)

Let $\mathcal{M}_{i}^{\prime}$ be the portion of $\mathcal{M}_{i}$ in one of the open sets, and let $\mathcal{M}_{i}^{\prime \prime}$ be the portion of $\mathcal{M}_{i}$ in the other. Each of these is a brakke flow (in $\mathbf{B}^{n+1,1}(0,3)$ ) so, by passing to a subsequence, we may assume that they converge to brakke flows $\mathcal{M}^{\prime}$ and $\mathcal{M}^{\prime \prime}$ in $\mathbf{B}^{n+1,1}(0,3)$. Now

$$
\operatorname{spt} \mathcal{M}_{i}^{\prime} \cup \operatorname{spt} \mathcal{M}_{i}^{\prime \prime} \rightarrow H \cap \mathbf{B}^{n+1,1}(0,3)
$$

and

$$
\text { HausDist }\left(\operatorname{spt} \mathcal{M}_{i}^{\prime}, \operatorname{spt} \mathcal{M}_{i}^{\prime \prime}\right) \leq 2 \tilde{R}_{i} \rightarrow 0
$$

so

$$
\operatorname{spt} \mathcal{M}^{\prime}=\operatorname{spt} \mathcal{M}^{\prime \prime}=H \cap \mathbf{B}^{n+1,1}(0,3) .
$$

Thus $\mathcal{M}^{\prime}$ and $\mathcal{M}^{\prime \prime}$ are (in $\mathbf{B}^{n+1,1}(0,3)$ ) a static plane with some multiplicities $m^{\prime}$ and $m^{\prime \prime}$. These multiplicities are integers, they are positive (by (6)), and they add up to 2 . Thus the multiplicities are each 1 . But now the brakke regularity theorem [B, 6.10] implies that the convergence of $\mathcal{M}_{i}^{\prime}$ and of $\mathcal{M}_{i}^{\prime \prime}$ is smooth on compact subsets of $\mathbf{B}^{n+1,1}(0,3)$.

8.4. Corollary. Suppose $\left(\mathcal{M}_{i}, \mathcal{K}_{i}\right)$ is a blow-up sequence converging to a limit flow $\left(\mathcal{M}^{\prime}, \mathcal{K}^{\prime}\right)$. Suppose one of the tangent flows to $\left(\mathcal{M}^{\prime}, \mathcal{K}^{\prime}\right)$ at a point $X$ is a static multiplicity 2 plane. Then there is a spacetime neighborhood $U$ of $X$ such that $\mathcal{K}^{\prime} \cap U$ is a smooth timelike hypersurface and such that (for large $\left.i\right),\left(\partial \mathcal{K}_{i}\right) \cap U$ consists of two components, each of which converges smoothly to $\mathcal{K}^{\prime} \cap U$.

Proof. By a suitable translation and dilation, we can assume that $X=0$ and that $\mathcal{K}^{\prime}$ satisfies the hypothesis of Theorem 8.1. The result follows immediately. 
The next corollary implies that no static multiplicity 2 planes occur as tangent flows to a mean-convex flow.

8.5. Corollary. Let $\left(\mathcal{M}^{\prime}, \mathcal{K}^{\prime}\right)$ be a tangent flow at a singular point $X$ of the original mean-convex flow $(\mathcal{M}, \mathcal{K})$, and suppose that there is a hyperplane $H$ and a $T \in$ $[0, \infty)$ such that $K_{t}^{\prime}=H$ for $-\infty<t \leq T$. Then $T=0$ and $K_{t}^{\prime}=\emptyset$ for $t>0$.

Proof. Suppose $T>0$. We can assume $X=0$ (otherwise translate by $-X$ ). We can also, by doing a suitable parabolic dilation, assume that $(\mathcal{M}, \mathcal{K})$ satisfies the hypothesis of Theorem 8.1. But then by 8.1, all the points in $\mathbf{B}^{n+1,1}(0,2)$ (including $X)$ are regular multiplicity 1 points, a contradiction.

8.6. Corollary. Let $\left(\mathcal{M}^{\prime}, \mathcal{K}^{\prime}\right)$ be a static or quasistatic limit flow to a mean-convex flow. Suppose $S$ is an $(n-1)$ cycle that bounds a rectifiable $n$-chain $Q$ in $\partial K_{-1}^{\prime}$. Then there is an $n$-dimensional rectifiable surface $V$ such that

(1) $\partial V=S$.

(2) No other surface bounded by $S$ has less area than $V$ does.

(3) $V$ is supported in $K_{-1}^{\prime}$.

Proof. Note that $M_{-1}^{\prime}$ is smooth at every point with a multiplicity 1 tangent plane (by Allard's regularity theorem applied to $M_{-t}^{\prime}$ or by Brakke's regularity theorem applied to the flow), and at every point with a multiplicity 2 tangent plane by Corollary 8.4. By Theorem 3.9, there are no tangent planes of higher multiplicity. Thus $M_{-1}^{\prime}$ is smooth almost everywhere. Now the proof of 8.6 is just like the proof of 6.1 , except that near points of multiplicity 2 we use 8.4 (instead of Brakke's regularity theorem) to get nice convergence.

8.7. Corollary. If $\left(\mathcal{M}^{\prime}, \mathcal{K}^{\prime}\right)$ is a static or quasistatic limit flow, then for $t<0$, $M_{t}^{\prime}$ is smooth except for a set of dimension $\leq n-7$.

Proof. The proof is just like the proof of 7.2, except that one uses Corollary 8.6 instead of Theorem 6.1, and one uses Corollary 8.4 in addition to the Brakke regularity theorem. (For the purposes of the proof, any points $X$ of $\mathcal{M}^{\prime}$ with a static multiplicity 2 tangent plane should also be regarded as regular points, which is reasonable in light of Corollary 8.4.)

\section{Quasistatic multiplicity two tangent Flows}

We have already shown that static multiplicity 2 planes do not occur as tangent flows for mean-convex surfaces. In this section, we rule out quasistatic tangent flows as well.

The following provides a convenient measure of how close a flow is to a quasistatic multiplicity 2 plane.

Definition. For $r>0$, let

$$
Q(r)=\mathbf{B}^{n+1}(0, r) \times\left(-r^{2}, 0\right)=\mathbf{B}^{n+1,1}(0, r) \cap\{(x, t): t<0\} .
$$

If $\mathcal{K}^{\prime}$ is a closed subset of spacetime, let $\phi\left(\mathcal{K}^{\prime}\right)$ be the infimum of $s>0$ such that

$$
\operatorname{HausDist}\left(\mathcal{K}^{\prime} \cap Q(1 / s), H \cap Q(1 / s)\right)<s
$$

for some static plane $H$ through the origin. 
Here HausDist $(A, B)$ is the Hausdorff distance between sets $A$ and $B$ : it is the infimum of $\epsilon>0$ such that every point in $A$ is within $\epsilon$ of some point of $B$ and such that every point in $B$ is within $\epsilon$ of some point of $A$.

9.1. Theorem. Let $(\mathcal{M}, \mathcal{K})$ be a mean-convex flow. There is an $\eta>0$ such that if $\left(\mathcal{M}^{\prime}, \mathcal{K}^{\prime}\right)$ is a tangent flow with $\phi\left(\mathcal{K}^{\prime}\right)<\eta$, then $\phi\left(\mathcal{K}^{\prime}\right)=0$.

Proof. Let $\left(\mathcal{M}_{i}, \mathcal{K}_{i}\right)$ be a sequence of tangent flows with

$$
\phi\left(\mathcal{K}_{i}\right) \rightarrow 0
$$

We must show that $\phi\left(\mathcal{K}_{i}\right)=0$ for all sufficiently large $i$.

By Theorem 8.2, associated with the $\mathcal{K}_{i}$ are functions $f_{i}$ and $g_{i}$ (defined on increasingly large subsets of $\left.\mathbf{R}^{n} \times(-\infty, 0)\right)$ such that:

(1) Either $f_{i}<g_{i}$ everywhere, or $f_{i} \equiv g_{i}$.

(2) The $f_{i}$ and $g_{i}$ converge uniformly and smoothly on compact subsets of $\mathbf{R}^{n} \times$ $(-\infty, 0)$ to 0 .

(3) The $f_{i}$ and $g_{i}$ are solutions of the non-parametric mean curvature flow equation.

(4) Given any $U \subset \subset \mathbf{R}^{n+1} \times(-\infty, 0)$, for sufficiently large $i, \mathcal{K}_{i}$ coincides in $U$ (after a rotation) with the region between the graphs of $f_{i}$ and $g_{i}$.

(5) $f_{i}(x, t)$ and $g_{i}(x, t)$ are increasing and decreasing, respectively, as functions of $t$.

Case 1: $f_{i}<g_{i}$ for infinitely many $i$.

By (3), the difference $u_{i}=g_{i}-f_{i}$ satisfies a linear parabolic equation. (See for example the proof of GT, 17.1] for the corresponding fact about elliptic equations.) The coefficients of the equation depend on $f_{i}$ and $g_{i}$, but tend smoothly to the coefficients of the ordinary heat equation as $i \rightarrow \infty$.

Thus for any $x \in \mathbf{R}^{n}, a<0$ and $r>0$, the Harnack inequality [L, VI.6.27] for parabolic equations implies that

$$
u_{i}(y, t) \leq C u_{i}(z, a)
$$

provided

$$
\begin{gathered}
y, z \in \mathbf{B}^{n}(x, r), \\
t \in\left[a-r^{2}, a\right)
\end{gathered}
$$

and provided $i$ is sufficiently large: $i \geq i(x, a, r)$.

Normally the parabolic Harnack inequality does not provide an upper bound for $u_{i}$ at one spacetime point in terms of $u_{i}$ at an earlier point. However, by (5), $u_{i}(x, t)$ is a decreasing function of $t$. Consequently, (6) implies that

$$
u_{i}(y, t) \leq C u_{i}(z, a) \leq C u_{i}(z, s)
$$

for any $s \in\left[a-r^{2}, a\right]$.

Now any compact subset $V$ of $\mathbf{R}^{n} \times(-\infty, 0)$ is contained in a subset of the form $\mathbf{B}^{n}(x, r) \times\left[a-r^{2}, a\right]$.

Thus (8) implies that

$$
\limsup _{i \rightarrow \infty} \sup _{Y, Z \subset V} \frac{u_{i}(Y)}{u_{i}(Z)} \leq C .
$$


Consequently the functions

$$
\tilde{u}_{i}:=\frac{u_{i}}{u_{i}(0,-1)}
$$

are uniformly bounded on compact subsets of $\mathbf{R}^{n} \times(-\infty, 0)$ as $i \rightarrow \infty$. The Schauder estimates $[\mathrm{L}, \mathrm{IV}]$ then imply that the various derivatives are also uniformly bounded on compact sets as $i \rightarrow \infty$. Thus a subsequence of the $\tilde{u}_{i}$ converges smoothly on compact sets to a function

$$
u: \mathbf{R}^{n} \times(-\infty, 0) \rightarrow \mathbf{R}
$$

such that

$$
u(0,-1)=1 .
$$

Note that $u$ is a positive solution of the standard heat equation, and that $u(z, t)$ is a decreasing function of $t$ for every $z$.

Let $w=u-\inf u$. Then $w$ is also a positive solution of the heat equation on $\mathbf{R}^{n} \times(-\infty, 0)$, and $w(z, t)$ is a decreasing function of $t$ for every $z$. By applying the Harnack inequality exactly as above, we see that

$$
\sup w \leq C \inf w .
$$

Since $\inf w=0$, this means $w \equiv 0$, and thus that $u \equiv 1$.

However, since $\left(\mathcal{M}_{i}, \mathcal{K}_{i}\right)$ is a tangent flow, it is self-similar for $t<0$, which means

$$
\begin{aligned}
f_{i}\left(r x, r^{2} t\right) & \equiv r f_{i}(x, t), \\
g_{i}\left(r x, r^{2} t\right) & \equiv r g_{i}(x, t)
\end{aligned}
$$

for $t<0$. This in turn implies that

$$
u\left(r x, r^{2} t\right) \equiv r u(x, t) \quad(t<0)
$$

which is a contradiction, since $u \equiv 1$. This proves that Case 1 does not occur.

Case 2: $f_{i} \equiv g_{i}$ for all sufficiently large $i$.

By (5), $f_{i}=g_{i}$ is both increasing and decreasing in $t$, and therefore must be independent of $t$ :

$$
f_{i}(x, t)=g_{i}(x, t)=h_{i}(x) .
$$

Combining this with $(*)$ gives

$$
h_{i}(r x) \equiv r h_{i}(x)
$$

for $r>0$. Since $h_{i}$ is smooth and homogeneous of degree 1 , it must in fact be linear. But this implies that $\left(\mathcal{M}_{i}, \mathcal{K}_{i}\right)$ is a multiplicity 2 plane, i.e., that $\phi\left(\mathcal{K}_{i}\right)=0$.

9.2. Theorem. A quasistatic multiplicity 2 plane cannot occur as a tangent flow to a mean-convex flow $(\mathcal{M}, \mathcal{K})$.

Proof. Suppose on the contrary that one of the tangent flows at a point $X$ is a quasistatic multiplicity 2 plane. By translating in spacetime, we may assume that $X=0$ (so the flow starts at some initial time $t_{0}<0$ ).

Let $\mathcal{D}_{\lambda} \mathcal{K}$ denote the result of parabolically dilating $\mathcal{K}$ by $\lambda$ (so that $(x, t) \mapsto$ $\left.\left(\lambda x, \lambda^{2} t\right)\right)$.

Note that the set of subsequential limits (as $\lambda \rightarrow \infty$ ) of

$$
\phi\left(\mathcal{D}_{\lambda} \mathcal{K}\right)
$$


is connected. (This is simply because $\lambda \mapsto \phi\left(\mathcal{D}_{\lambda} \mathcal{K}\right)$ is continuous.) Now by hypothesis, 0 is one subsequential limit. By Theorem 9.1, no subsequential limits lie in the interval $(0, \eta)$. Thus 0 is the only subsequential limit:

$$
\lim _{\lambda \rightarrow \infty} \phi\left(\mathcal{D}_{\lambda} \mathcal{K}\right)=0 .
$$

That is, all the tangent flows at 0 are quasistatic multiplicity 2 planes.

Now let

$$
V(r)=\sup \left\{\rho: 0 \in \mathbf{B}^{n+1}(z, \rho) \subset K_{-r^{2}} \text { for some } z\right\} .
$$

(Intuitively, at time $t=-r^{2}, \partial K_{t}$ consists, near the origin, of two sheets: $V(r)$ measures how far apart the two sheets are at the origin.)

Then by (1)

$$
\lim _{r \rightarrow 0} \frac{V(r)}{r}=0 .
$$

Thus in particular there must be a sequence $r(i) \rightarrow 0$ such that

$$
\frac{V(r(i))}{r(i)}<2 \frac{V(3 r(i))}{3 r(i)} .
$$

Just as in the proof of Theorem 9.1, we apply Theorem 8.2 to $\mathcal{D}_{1 / r(i)} \mathcal{K}$ to get functions $f_{i}$ and $g_{i}$ such that

(1) Either $f_{i}<g_{i}$ everywhere, or $f_{i} \equiv g_{i}$.

(2) The $f_{i}$ and $g_{i}$ converge uniformly and smoothly on compact subsets of $\mathbf{R}^{n} \times$ $(-\infty, 0)$ to 0 .

(3) The $f_{i}$ and $g_{i}$ are solutions of the non-parametric mean curvature flow equation.

(4) Given any $U \subset \subset \mathbf{R}^{n+1} \times(-\infty, 0)$, for sufficiently large $i, \mathcal{D}_{1 / r(i)} \mathcal{K}$ coincides in $U$ (after a rotation) with the region between the graphs of $f_{i}$ and $g_{i}$.

As in the proof of the lemma, we can choose $c_{i}>0$ such that

$$
c_{i}\left(g_{i}-f_{i}\right)
$$

subsequentially converges smoothly on compact sets to the constant function $u(x, t)$ $\equiv 1$ on $\mathbf{R}^{n} \times(-\infty, 0)$.

Note that

$$
V(\lambda r(i)) \sim g_{i}\left(0,-\lambda^{2}\right)-f_{i}\left(0,-\lambda^{2}\right),
$$

so (3) implies that

$$
\frac{u(0,-1)}{1} \leq 2 \frac{u(0,-9)}{3} .
$$

But this contradicts the fact that $u \equiv 1$.

\section{Proof OF THE MAIN THEOREM}

To complete the proof of Theorem 1.1, note that Theorem 3.9 excludes static and quasistatic planes of multiplicity $>2$ as tangent flows. Corollary 8.5 and Theorem 9.2 exclude static and quasistatic planes, respectively, of multiplicity 2. Corollary 8.7 excludes all static or quasistatic polyhedral cones. Thus by the stratification theorem 1.3, the singular set has parabolic dimension $\leq n-1$. 


\section{OTHER AMBIENT MANIFOLDS}

The main theorem 1.1 remains true when $\mathbf{R}^{n+1}$ is replaced by an arbitrary smooth riemannian manifold. Only minor, straightforward modifications are needed in the proofs. However, the long-term behavior can be different. In particular, any compact set in euclidean space vanishes in a finite time under the level set flow, but there are two other possibilities in other ambient manifolds.

Consider for example a smooth compact embedded strictly stable minimal hypersurface $S$ in a riemannian manifold $N$. Suppose that $S$ is the boundary of a region $W$ with $W \cup S$ compact. (For example, $N$ could be a pair of two-spheres joined by a thin tube, and $S$ could be a closed geodesic going around the tube.) The strict stability implies that there is a tubular neighborhood $K$ of $S$ such that $K$ is mean-convex. Then $F_{t}(K)$ converges to $S$ as $t \rightarrow \infty$. Indeed, the boundary of $F_{t}(K)$ consists of two components, one on either side of $S$, and each component converges smoothly to $S$ as $t \rightarrow \infty$.

Now let $K^{\prime}=K \cup W$. Then $K^{\prime}$ is also mean-convex. Now $F_{t}\left(K^{\prime}\right)$ converges to $W \cup S$, and the boundary of $F_{t}\left(K^{\prime}\right)$ has only one connected component, which converges smoothly to $S$ as $t \rightarrow \infty$.

These examples illustrate the following theorem:

11.1. Theorem. Let $K$ be a compact, mean-convex subset of a smooth riemannian $(n+1)$-dimensional manifold $N$. Let $F_{t}(K)$ be the result of flowing $K$ for time $t$ by the level set flow. Let

$$
K_{\infty}=\bigcap_{t} F_{t}(K)
$$

Then $K_{\infty}$ has finitely many connected components. The boundary of each component $C$ is a stable minimal variety whose singular set has hausdorff dimension $\leq n-7$.

The boundary of $F_{t}(K)$ converges smoothly to $\partial K_{\infty}$ away from the singular set of $\partial K_{\infty}$. (If a component $C$ has interior points, then the convergence is locally "one-sheeted"; if not, the convergence is locally two-sheeted.)

Proof. Let $(\mathcal{M}, \mathcal{K})$ be the original flow. Let

$$
\left(\mathcal{M}^{T}, \mathcal{K}^{T}\right)
$$

be the result of translating $(\mathcal{M}, \mathcal{K})$ by $(x, t) \mapsto(x, t-T)$. Then a subsequence will converge to a limit $\left(\mathcal{M}^{\prime}, \mathcal{K}^{\prime}\right)$. Note that

$$
\mathcal{K}^{\prime}=\left(\bigcap_{t} K_{t}\right) \times \mathbf{R}
$$

This is independent of the sequence of $T^{\prime}$ 's going to infinity. Also, as in the remark at the end of $\S 5, \mathcal{M}^{\prime}$ is determined by $\mathcal{K}^{\prime}$. Thus

$$
\left(\mathcal{M}^{T}, \mathcal{K}^{T}\right) \rightarrow\left(\mathcal{M}^{\prime}, \mathcal{K}^{\prime}\right)
$$

as $T \rightarrow \infty$. (In other words, it is not necessary to pass to a subsequence.)

Note by $(*)$ that the flow $\left(\mathcal{M}^{\prime}, \mathcal{K}^{\prime}\right)$ is static:

$$
\begin{aligned}
M_{t}^{\prime} & =M^{\prime}, \\
K_{t}^{\prime} & =K^{\prime}\left(=K_{\infty}\right) .
\end{aligned}
$$

Thus by Corollary $8.7, \partial K_{\infty}$ is a stable minimal hypersurface with singular set of dimension $\leq n-7$. 
Finally, the smooth convergence follows from Brakke's regularity theorem B. 6.10] (at points of multiplicity 1 in $M^{\prime}$ ) and from the sheeting theorem 8.1 at points of multiplicity 2 in $M^{\prime}$. (By the multiplicity bound theorem 3.9, no points of $M^{\prime}$ have tangent planes with multiplicity $>2$.)

\section{Multiplicity 2 Limit Flows}

We have shown that multiplicity 2 planes do not occur as tangent flows to meanconvex flows. That leaves open the possibility that a multiplicity 2 plane might occur as a limit flow to a mean-convex flow. Here we show that this possibility does not occur in low dimensions. This fact will be used in [W5] to show that in low dimensions the only tangent flows that occur are shrinking cylinders.

12.1. Theorem. Let $\left(\mathcal{M}^{\prime}, \mathcal{K}^{\prime}\right)$ be a limit flow to a mean-convex flow. Suppose one of the tangent flows to $\left(\mathcal{M}^{\prime}, \mathcal{K}^{\prime}\right)$ at $X=(x, t)$ is a static or quasistatic multiplicity 2 plane. Then there is a spatial neighborhood $W$ of $x$, a minimal properly embedded smooth minimal surface $S$ in $w$, and an interval $(a, b)$ (with $a<t)$ such that

$$
K_{\tau}^{\prime} \cap W=S \quad \forall \tau \in(a, b) .
$$

If the tangent flow is static, we may take $b>t$.

Remark. One can actually show that there is a $T \geq t$ (possibly infinite) such that

$$
K_{\tau}^{\prime} \cap W= \begin{cases}S & \text { if } \tau \leq T, \\ \emptyset & \text { if } \tau>T,\end{cases}
$$

but we do not need this fact.

Proof. The static case has already been proven (8.4).

Now in the quasistatic case, we proceed exactly as in the proof of 9.2. As in that proof, we get a sequence of functions $f_{i}$ and $g_{i}$. If $f_{i}<g_{i}$, then we get a contradiction as in 9.2.

If this is not the case for some $i$, then $f_{i} \equiv g_{i}$. But that immediately gives (1) (for some $a$ and $b$ with $a<b<t$ ).

12.2. Theorem. Under the hypotheses of Theorem 12.1,

$$
\Theta\left(\mathcal{M}^{\prime}, \cdot\right) \geq 2
$$

on all of $S \times(-\infty, b]$.

Proof. Let $\mathcal{Z}$ be the set of points of $\mathcal{M}^{\prime}$ at which none of the tangent flows is a static or quasistatic plane. A tangent flow to $\mathcal{M}^{\prime}$ at any point of $\mathcal{Z}$ is also a limit flow to $\mathcal{M}$, and therefore cannot be a static or quasistatic polyhedral cone (by 8.7 ). The stratification theory W3 then implies that $\mathcal{Z}$ has parabolic hausdorff dimension $\leq n-1$.

Let $\pi(\mathcal{Z})$ be the spatial projection of $\mathcal{Z}$ :

$$
\pi(\mathcal{Z})=\bigcup_{\tau}\{y:(y, \tau) \in \mathcal{Z}\}
$$

Then $\pi(\mathcal{Z})$ has hausdorff dimension $\leq n-1$, so almost every $y \in S$ does not belong to $\pi(\mathcal{Z})$. Fix such a $y$.

We claim that $\Theta\left(\mathcal{M}^{\prime},(y, \tau)\right)=2$ for all $\tau<b$. For if not, let

$$
T^{*}=\sup \left\{\tau<b: \Theta\left(\mathcal{M}^{\prime},(y, \tau)\right) \neq 2\right\} .
$$


Of course $T^{*} \leq a$ by 1 . Let $\left(\mathcal{M}^{\prime \prime}, \mathcal{K}^{\prime \prime}\right)$ be a tangent flow to $\left(\mathcal{M}^{\prime}, \mathcal{K}^{\prime}\right)$ at $\left(y, T^{*}\right)$. Note that $\left(\mathcal{M}^{\prime \prime}, \mathcal{K}^{\prime \prime}\right)$ cannot be a quasistatic plane since $\Theta\left(\mathcal{M}^{\prime},(y, \tau)\right)=2$ for $T^{*} \leq \tau<b$. For the same reason, $\left(\mathcal{M}^{\prime \prime}, \mathcal{K}^{\prime \prime}\right)$ cannot be a static multiplicity 1 plane. It also cannot be a static multiplicity 2 plane, since Theorem 12.1 would then force $\Theta\left(\mathcal{M}^{\prime}, \cdot\right)$ to be 2 on a spacetime neighborhood of $\left(y, T^{*}\right)$, contradicting the definition of $T^{*}$.

We have shown that $\left(\mathcal{M}^{\prime \prime}, \mathcal{K}^{\prime \prime}\right)$ cannot be a static or quasistatic plane. Thus $\left(y, T^{*}\right) \in \mathcal{Z}$. But this contradicts the choice of $y$, proving the claim.

So we have shown for almost every $y \in S$ (namely for every $y \in S \backslash \pi(\mathcal{Z})$ ) that

$$
\Theta\left(\mathcal{M}^{\prime},(y, \tau)\right)=2 \quad \text { for } \tau<b .
$$

By upper semicontinuity of $\Theta, \Theta\left(\mathcal{M}^{\prime}, \cdot\right) \geq 2$ on all of $S \times(-\infty, b]$.

12.3. Theorem. Let $(\mathcal{M}, \mathcal{K})$ be a mean-convex flow. Suppose one of the limit flows to $(\mathcal{M}, \mathcal{K})$ at $X$ is a static or quasistatic multiplicity 2 plane. Then a static non-planar minimal cone also occurs as a limit flow at $X$.

Proof. Let $\left(\mathcal{M}_{i}, \mathcal{K}_{i}\right)$ be the blow up sequence converging to a static or quasistatic multiplicity 2 plane. We may assume (by making slight translation, if necessary) that the spacetime origin is in $\partial \mathcal{K}_{i}$ for every $i$.

Let $R_{i}$ be the smallest $R \geq 1$ for which

$$
\phi\left(\mathcal{K}_{i}^{\prime}\right) \geq \eta / 2
$$

where $\mathcal{K}_{i}^{\prime}$ is obtained by dilating $\mathcal{K}_{i}$ parabolically by $1 / R_{i}$. (Here $\phi$ and $\eta$ are as in Theorem 9.1.) Note that $R_{i} \rightarrow \infty$ by hypothesis. Passing to a subsequence if necessary, we may assume that the $\left(\mathcal{M}_{i}^{\prime}, \mathcal{K}_{i}^{\prime}\right)$ converge to a limit $\left(\mathcal{M}^{\prime}, \mathcal{K}^{\prime}\right)$.

Note that

$$
\phi\left(\mathcal{K}^{\prime}\right) \geq \eta / 2
$$

but

$$
\phi\left(\mathcal{D}_{\lambda} \mathcal{K}^{\prime}\right) \leq \eta / 2 \quad \forall \lambda>1 .
$$

By (2) and Theorem 9.1, every tangent flow to $\left(\mathcal{M}^{\prime}, \mathcal{K}^{\prime}\right)$ at the origin (of spacetime) is a static or quasistatic multiplicity 2 plane.

(Note this means that $\left(\mathcal{M}^{\prime}, \mathcal{K}^{\prime}\right)$ is not just a homothetic image of the original flow $(\mathcal{M}, \mathcal{K})$, since the original flow has no multiplicity 2 static or quasistatic planes as tangent flows $(9.2,8.5)$. Thus $\left(\mathcal{M}^{\prime}, \mathcal{K}^{\prime}\right)$ must indeed be a limit flow of $(\mathcal{M}, \mathcal{K})$.)

By Theorem 12.2, there is a $b \in \mathbf{R}$ and a minimal hypersurface $S$ containing the origin such that

$$
\Theta\left(\mathcal{M}^{\prime}, \cdot\right) \geq 2 \text { on } S \times(-\infty, b] .
$$

Now translate $\left(\mathcal{M}^{\prime}, \mathcal{K}^{\prime}\right)$ by $(x, t) \mapsto(x, t+j)$ and then let $j$ tend to infinity to get a static limit flow $\left(\mathcal{M}^{\prime \prime}, \mathcal{K}^{\prime \prime}\right)$ with

$$
K^{\prime \prime}=K_{t}^{\prime \prime}=\bigcup_{\tau} K_{\tau}^{\prime} \quad \forall t .
$$

Now by (3),

$$
\Theta\left(\mathcal{M}^{\prime \prime}, \cdot\right) \geq 2 \text { on } S \times \mathbf{R} .
$$

By (1), $K^{\prime \prime}$ is not a plane, which (by monotonicity) means that the density at infinity of $M^{\prime \prime}$ is $>2$. 
Let $\left(\mathcal{M}^{*}, \mathcal{K}^{*}\right)$ be a tangent flow at infinity to $\left(\mathcal{M}^{\prime \prime}, \mathcal{K}^{\prime \prime}\right)$ (that is, a subsequential limit as $\lambda \rightarrow 0$ of $\left.\mathcal{D}_{\lambda}\left(\mathcal{M}^{\prime \prime}, \mathcal{K}^{\prime \prime}\right)\right)$. Then $\left(\mathcal{M}^{*}, \mathcal{K}^{*}\right)$ is a static minimal cone of density $>2$. If the cone were a plane, then by 5.4 the multiplicity of the plane would be 1 or 2. Thus the cone $M^{*}$ is not planar.

12.4. Corollary. If $n<7$, then no static or quasistatic multiplicity 2 plane occurs as a limit flow to a mean-convex flow in $\mathbf{R}^{n+1}$ (or in an $(n+1)$-dimensional riemannian manifold).

Proof. By 8.7, no non-planar cones occur as limit flows for $n<7$.

12.5. Corollary. If $n<7$, then the "grim reaper flow"

$$
\mathcal{K}^{\prime}=\left\{(x, t): x_{2} \geq t-\ln \cos x_{1},-\pi / 2<x_{1}<\pi / 2\right\}
$$

does not occur as a limit flow to any mean-convex flow in $\mathbf{R}^{n+1}$.

Proof. The tangent flow at infinity to the grim reaper flow is a quasistatic multiplicity 2 plane. Since the latter does not occur as a limit flow to a mean-convex flow (by 12.4), neither can the former (by 5.2(1)).

\section{REFERENCES}

[B] K. Brakke, The motion of a surface by its mean curvature, Princeton Univ. Press, 1978. MR 82c: 49035

[CGG] Y. G. Chen, Y. Giga, and S. Goto, Uniqueness and existence of viscosity solutions of generalized mean curvature flow equations, J. Diff. Geom. 33 (1991), 749-786. MR 93a:35093

[ES] L. C. Evans and J. Spruck, Motion of level sets by mean curvature I, J. Diff. Geom. 33 (1991), 635-681; II, Trans. Amer. Math. Soc. 330 (1992), 321-332; III, J. Geom. Anal. 2 (1992), 121-150; IV, J. Geom. Anal. 5 (1995), 77-114. MR 92h:35097, MR 92f:58050. MR 93d:58044 IMR 96a:35077

[F] H. Federer, The singular sets of area minimizing rectifiable currents with codimension one and of area minimizing flat chains modulo two with arbitrary codimension, Bull. Amer. Math. Soc. 76 (1970), 767-771. MR 41:5601

[GT] D. Gilbarg and N. Trudinger, Elliptic partial differential equations of second order, 2nd edition, Springer, New York, 1983. MR 86c:35035

[H1] G. Huisken, Lecture one: mean curvature evolution of closed hypersurfaces, Tsing Hua lectures on geometry and analysis (Hsinchu, 1990-1991), Internat. Press, Cambridge, MA, 1997, pp. 117-123; Lecture two: singularities of the mean curvature flow, pp. 125-130. MR 99a:53040

[H2] Asymptotic behavior for singularities of the mean curvature flow, J. Diff. Geom. 31 (1990), 285-299. MR 90m:53016

[H3] Local and global behavior of hypersurfaces moving by mean curvature, Proc. Sympos. Pure Math. 54 (1993), 175-191. MR 94c:58037

[HS] G. Huisken and C. Sinestrari, Convexity estimates for mean curvature flow and singularities of mean convex surfaces, Acta Math. 183 (1999), 45-70. CMP 2000:05

[I1] T. Ilmanen, Elliptic regularization and partial regularity for motion by mean curvature, Mem. Amer. Math. Soc. 108 (520) (1994). MR 95d:49060

[I2] Generalized flow of sets by mean curvature on a manifold, Indiana Univ. Math. J. 41 (1992), 671-705. MR 93k:58057

[I3] The level-set flow on a manifold, Proc. Sympos. Pure Math. 54 (1993), 193-204. MR 94d:58040

[I4] Singularities of mean curvature flow of surfaces, preprint.

[L] G. Lieberman, Second order parabolic differential equations, World Scientific, Singapore, 1996. MR 98k:35003

[OS] S. Osher and J. Sethian, Fronts propagating with curvature-dependent speed: algorithms based on Hamilton-Jacobi formulations, J. Comput. Phys. 79 (1988), 12-49. MR 89h:80012 
[SJ] J. Simons, Minimal varieties in riemannian manifolds, Ann. of Math. 88 (1962), 62-105. MR 38:1617

[SL] L. Simon, Lectures on geometric measure theory, Proc. Centre for Math. Analysis 3 (1983). MR 87a:49001

[SS] R. Schoen and L. Simon, Regularity of stable minimal hypersurfaces, Comm. Pure Appl. Math. 34 (1981), 741-797. MR 82k:49054

[St] A. Stone, A density function and the structure of singularities of the mean curvature flow, Calc. Var. Partial Diff. Equations 2 (1994). MR 97c:58030

[W1] B. White, Partial regularity of mean-convex hypersurfaces flowing by mean curvature, International Math. Research Notices 4 (1994), 185-192. MR 95b:58042

[W2] The topology of hypersurfaces moving by mean curvature, Comm. Analysis and Geom. 3 (1995), 317-333. MR 96k:58051

[W3] Stratification of minimal surfaces, mean curvature flows, and harmonic maps, J. reine angew. Math. 488 (1997), 1-35. MR 99b:49038

[W4] A local regularity theorem for classical mean curvature flow, preprint.

[W5] - The nature of singularities in mean curvature flow of mean-convex surfaces, preprint (available at http://math.stanford.edu/ ${ }^{\sim}$ white).

[W6] - Subsequent singularities in mean curvature flow of mean convex surfaces, in preparation.

Department of Mathematics, Stanford University, Stanford, California 94305

E-mail address: white@math.stanford.edu 